\title{
Approximation of fractals by discrete graphs: norm resolvent and spectral convergence
}

\author{
Olaf Post and Jan Simmer \\ Fachbereich 4 - Mathematik, Universität Trier, 54286 Trier, Germany \\ E-mail address: olaf.post@uni-trier.de \\ Fachbereich 4 - Mathematik, Universität Trier, 54286 Trier, Germany \\ E-mail address: simmer@uni-trier.de
}

\begin{abstract}
We show a norm convergence result for the Laplacian on a class of pcf self-similar fractals with arbitrary Borel regular probability measure which can be approximated by a sequence of finite-dimensional weighted graph Laplacians.

As a consequence other functions of the Laplacians (heat operator, spectral projections etc.) converge as well in operator norm. One also deduces convergence of the spectrum and the eigenfunctions in energy norm.
\end{abstract}

\section{Introduction}

The aim of this article is to apply the abstract concept of convergence of energy forms defined on different Hilbert spaces, developed by the first author in [Pos12, Pos06] to the case of certain self-similar fractals. More precisely, this concept of convergence of energy forms is based on quasi-unitary equivalence, a (quantitative) generalisation of unitary equivalence and norm resolvent convergence in the sense of Remark 2.2 (see Section 2 for further details). We also give some consequences of quasi-unitary equivalence in Corollaries 1.2 1.4 below. Details on self-similar fractals and precise definitions can be found in Kig01, Str06] going back to Kig93a, Kig93b; see also Section 3 where we give an informal presentation.

A self-similar set $K \subset \mathbb{R}^{d}$ is generated by contractive similarities $F_{1}, \ldots, F_{N}: \mathbb{R}^{d} \longrightarrow \mathbb{R}^{d}$ such that

$$
K=\bigcup_{j=1, \ldots, N} F_{j}(K)
$$

Our analysis works for a class of pcf self-similar sets (see Definition 3.1) which we call here fractals approximable by finite weighted graphs (see Definition 3.4). For such a fractal $K$ there is a sequence $\left(G_{m}\right)_{m \in \mathbb{N}_{0}}$ of nested graphs $G_{m}=\left(V_{m}, E_{m}\right)$ (i.e., $V_{m} \subset V_{m+1} \subset K$ ) and conductances (i.e., edge weights) $c_{e, m}>0$ of the edges $e \in E_{m}$, such that $V_{*}=\bigcup_{m} V_{m}$ is dense in $K$, (see e.g. Figure 1 for the pentagasket with all five fixed points as boundary vertices) together with a compatible and self-similar sequence of graph energies $\left(\mathcal{E}_{m}\right)_{m}$ (see Definitions 3.2 and 3.3 ) given by

$$
\mathcal{E}_{m}(f):=\sum_{e=\{x, y\} \in E_{m}} c_{e, m}|f(x)-f(y)|^{2}
$$

for $f: V_{m} \longrightarrow \mathbb{C}$.

The compatibility roughly means that $\mathcal{E}_{m}(\varphi)$ agrees with the energy of $\mathcal{E}_{m+1}(h)$ where $h: V_{m+1} \longrightarrow \mathbb{C}$ is the harmonic extension of $\varphi: V_{m} \longrightarrow \mathbb{C}$. The harmonic extension $h$ has the property that it minimises the energy $\mathcal{E}_{m}(u)$ among all extensions $u: V_{m+1} \longrightarrow \mathbb{C}$ with $u \uparrow_{V_{m}}=\varphi$, see 3.2 .

Date: September 10, 2018, 1:37, File: fractal-approx-graphs.v7.tex. 

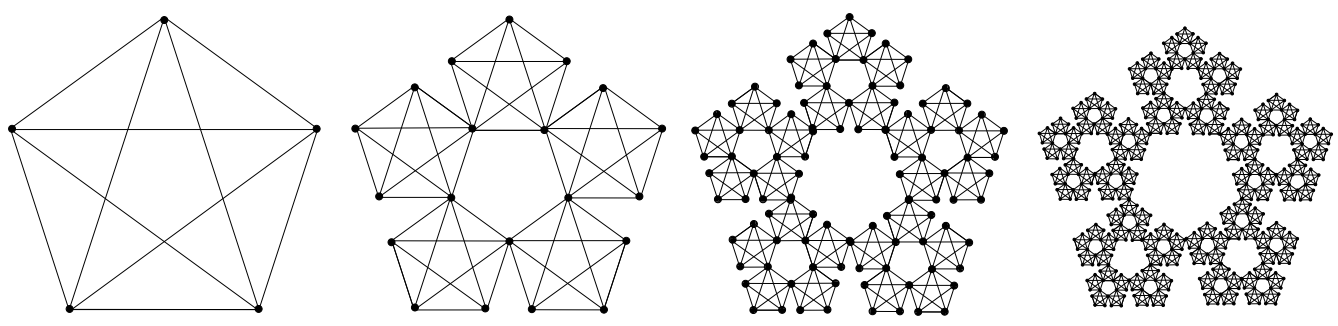

FiguRE 1. The pentagasket approximation graphs $G_{m}$ starting with all five fixed points as boundary $V_{0}$ for the generations $m=0, \ldots, 3$ with $\left(\left|V_{m}\right|\right)_{m}=$ $(5,5 \cdot 5-5=20,5 \cdot 20-5=95,95 \cdot 5-5=470, \ldots)$.

Self-similarity means that $\mathcal{E}_{m+1}$ can be constructed from $\mathcal{E}_{m}$ together with the renormalisation factors and contractive similarities defining the fractal $K$, see (3.4).

The existence of a compatible and self-similar sequence $\left(\mathcal{E}_{m}\right)_{m}$ (also referred to as the renormalisation problem, see Remark 3.5 is not guaranteed for a general pcf fractal, but the class of such fractals is sufficiently large to give an interesting theory. In particular, the Sierpiński gasket, its higher-dimensional analogs, the pentagasket and the hexagasket (resp. any $n$-gasket if $n$ is not divisible by 4) all belong to the class of fractals approximable by finite weighted graphs.

The compatibility of $\left(\mathcal{E}_{m}\right)_{m}$ guarantees that the limit

$$
\widetilde{\mathcal{E}}(u):=\lim _{m \rightarrow \infty} \mathcal{E}_{m}\left(u \uparrow_{V_{m}}\right)
$$

exists for continuous functions $u: K \longrightarrow \mathbb{C}$ (and may be $\infty$ ). The domain of $\widetilde{\mathcal{E}}$ consists of those continuous functions for which the limit is finite. We call the non-negative closed quadratic form $\widetilde{\mathcal{E}}$ the energy form of the fractal $K$ (and the sequence of approximating graphs $\left(G_{m}\right)_{m}$ ).

We fix a Borel regular probability measure $\mu$ on $K$ having full support (e.g. the homogeneous and self-similar measure associating $\mu\left(F_{j}(K)\right)=1 / N$ to each self-similar image of $K$ or the Kusuoka energy measure, see Subsection 5.1). On each approximating graph $G_{m}$, we define a discrete probability measure $\mu_{m}$, i.e., a family $\left(\mu_{m}(x)\right)_{x \in V_{m}}$ as the integral of the $m$-harmonic function with boundary value 1 at $x \in V_{m}$ and 0 at $V_{m} \backslash\{x\}$ (see (3.13); for a self-similar measure, $\mu_{m}(x)$ decays exponentially in $\left.m\right)$. We call the family $\left(\mu_{m}\right)_{m}$ of probability measures on $G_{m}$ the approximating measures corresponding to $(K, \mu)$.

Remark. We would like to stress that the definitions of energy forms and harmonic functions on $K$ and $G_{m}$ do not refer to a measure. But once we are interested in the corresponding operators, eigenvalues or convergence results (as explained below), we need to fix a measure $\mu$ and choose for $\left(\mu_{m}\right)_{m}$ the approximating measures corresponding to $(K, \mu)$ as above.

Let $\widetilde{\mathscr{H}}=\mathrm{L}_{2}(K, \mu)$ and $\mathscr{H}_{m}=\ell_{2}\left(V_{m}, \mu_{m}\right)$ be the corresponding Hilbert spaces, respectively. Denote by $\widetilde{\Delta}$ resp. $\Delta_{m}$ the operators on the fractal resp. the discrete graph associated with the energy forms $\widetilde{\mathcal{E}}$ resp. $\mathcal{E}_{m}$ in $\widetilde{\mathscr{H}}$ resp. $\mathscr{H}_{m}$. The graph operator can actually be represented as a matrix of increasing size, as the graphs $G_{m}$ are finite.

For a fractal approximable by finite weighted graphs, the spectrum of $\widetilde{\Delta}$ is purely discrete and hence consists of a sequence of eigenvalues $\left(\lambda_{k}(\widetilde{\Delta})\right)_{k \in \mathbb{N}}$ in increasing order and repeated according to the multiplicity.

\subsection{Main results}

Our first main result is the statement that the energy forms on $\left(G_{m}, \mu_{m}\right)$ and $(K, \mu)$ are $\delta_{m}$-quasi-unitarily equivalent with $\delta_{m} \rightarrow 0$ as $m \rightarrow \infty$. The $\delta_{m}$-quasi-unitary equivalence is formulated for quadratic forms acting in different Hilbert spaces and it implies a variant of norm resolvent convergence for the associated Laplacians. Our method does not rely on the monotonicity of the quadratic forms: It is well-known that an increasing sequence of quadratic 
forms on a given Hilbert space $\mathscr{H}$ converges to a limit form (see e.g. [RS80, Thm. S.14]), and that the corresponding operators converge in strong resolvent sense. Here, we face a problem as the quadratic forms $\mathcal{E}_{m}$ act in $\mathscr{H}_{m}$, and they are different for each $m \in \mathbb{N}$. Moreover, also the limit space $\widetilde{\mathscr{H}}$ is different, and it is a priori not clear how to relate these spaces. We comment on other approaches like Mosco convergence of forms in Subsection 1.2.

The proof of the following first main result will be given in Subsection 4.1:

1.1. Theorem. Let $(K, \mu)$ be a fractal approximable by finite weighted graphs $\left(G_{m}, \mu_{m}\right)$ with Borel regular probability measure $\mu$ of full support $K$. Then the fractal energy form $\mathcal{E}_{K}$ and the approximating graph energy forms $\mathcal{E}_{m}$ are $\delta_{m}$-quasi-unitarily equivalent on $\widetilde{\mathscr{H}}=\mathrm{L}_{2}(K, \mu)$ and $\mathscr{H}_{m}=\ell_{2}\left(G_{m}, \mu_{m}\right)$, where $\delta_{m} \rightarrow 0$ as $m \rightarrow \infty$ is given precisely in 4.3). In particular, $\delta_{m}$ decays exponentially fast.

The definition of quasi-unitary equivalence needs an identification operator

$$
J=J_{m}: \mathscr{H}_{m}=\ell_{2}\left(V_{m}, \mu_{m}\right) \longrightarrow \widetilde{\mathscr{H}}=\mathrm{L}_{2}(K, \mu)
$$

such that the resolvent weighted operator norms fulfil

$$
\left\|\left(\operatorname{id}_{\mathscr{H}_{m}}-J^{*} J\right)\left(\Delta_{m}+1\right)^{-1 / 2}\right\| \leq \delta_{m} \quad \text { and } \quad\left\|\left(\operatorname{id}_{\widetilde{\mathscr{H}}}-J J^{*}\right)(\widetilde{\Delta}+1)^{-1 / 2}\right\| \leq \delta_{m},
$$

hence tend to 0 . We usually suppress the dependence of $J$ on $m$ in the notation; in particular, the second operator norm also depends on $m$. The definition of quasi-unitary equivalence (Definition 2.1) is more elaborated, as it uses also identification operators on the form domain level and is expressed entirely in terms of the energy forms. On the operator level, $\delta_{m}$-quasiunitary equivalence implies that

$$
\left\|(\widetilde{\Delta}+1)^{-1} J-J\left(\Delta_{m}+1\right)^{-1}\right\| \leq \delta_{m}
$$

see [Pos12, Prp. 4.4.15]. Once quasi-unitary equivalence and hence $1.3 \mathrm{a})-(1.3 \mathrm{~b}$ ) are fulfilled, we can mimic the proofs of results following from norm resolvent convergence (which actually is the convergence of the norm $\left(1.3 \mathrm{~b}\right.$ to 0 for $\widetilde{\mathscr{H}}=\mathscr{H}_{m}$ and $\left.J=\mathrm{id}\right)$. These consequences are treated systematically in [Pos12, Ch. 4]; we just cite two of them here (see [Pos12, Thm. 4.2.14 and Thm. 4.3.5]):

The first consequence states in our setting that a function of the operator on the fractal is close to the corresponding operator on the graph sandwiched by $J^{*}$ and $J$ and vice versa:

1.2. Corollary. Let $\eta:[0, \infty) \longrightarrow \mathbb{C}$ be a function continuous in a neighbourhood $U$ of $\sigma(\widetilde{\Delta})$ such that $\lim _{\lambda \rightarrow \infty}(\lambda+1)^{1 / 2} \eta(\lambda)$ exists. Then there is a function $\delta \rightarrow \varepsilon(\delta)$ such that $\varepsilon(\delta) \rightarrow 0$ as $\delta \rightarrow 0$, depending only on $\eta$ and $U$, and

$$
\left\|\eta(\widetilde{\Delta})-J \eta\left(\Delta_{m}\right) J^{*}\right\| \leq \varepsilon\left(\delta_{m}\right) \quad \text { and } \quad\left\|\eta\left(\Delta_{m}\right)-J^{*} \eta(\widetilde{\Delta}) J\right\| \leq \varepsilon\left(\delta_{m}\right) .
$$

For a certain class of functions $\eta$, we can actually show that $\varepsilon$ is Lipschitz, i.e., that $\varepsilon(\delta)=$ $C_{\eta, U} \delta$. For example, $\eta(\lambda)=\mathrm{e}^{-t \lambda}$ or $\eta=\mathbb{1}_{I}$ with $\partial I \cap \sigma(\widetilde{\Delta})=\emptyset$ belong to this class. In particular, Corollary 1.2 then states the norm convergence of the approximating heat operators on the discrete graphs to the heat operators on the fractal. If $\eta=\mathbb{1}_{I}$ then we have the following statement about the spectral projections:

1.3. Corollary. Let $I$ be an interval in $\mathbb{R}$ such that $\partial I \cap \sigma(\widetilde{\Delta})=\emptyset$. Then the spectral projections converge in operator norm, i.e.,

$$
\left\|\mathbb{1}_{I}(\widetilde{\Delta})-J \mathbb{1}_{I}\left(\Delta_{m}\right) J^{*}\right\| \leq C_{I} \delta_{m}
$$

where the constant $C_{I}$ depends only on $I$ and the distance of $\partial I$ to $\sigma(\widetilde{\Delta})$.

As in the case of usual operator norm convergence, the operator norm convergence of spectral projections as in Corollary 1.3 also implies the convergence of eigenvalues (we give a direct proof in Theorem 4.4 using the min-max characterisation of eigenvalues in Proposition 2.8, under slightly stronger assumptions). Such an eigenvalue convergence result is known as folklore, at least for fractals with self-similar measure, and where the spectral decimation method is 
available (see e.g. [FS92]), but seems to be new in other cases or if the measure is not selfsimilar.

1.4. Corollary. Let $\lambda_{k}\left(\Delta_{m}\right)$ resp. $\lambda_{k}(\widetilde{\Delta})$ be the $k$-th eigenvalue of $\Delta_{m}$ resp. $\widetilde{\Delta}$ (in increasing order, repeated according to multiplicity). Then

$$
\left|\lambda_{k}\left(\Delta_{m}\right)-\lambda_{k}(\widetilde{\Delta})\right| \leq C_{k} \delta_{m}
$$

for all $m \in \mathbb{N}$ such that $\operatorname{dim} \mathscr{H}_{m} \geq k$, where $C_{k}$ depends only on $\lambda_{k}(\widetilde{\Delta})$ and where $\delta_{m} \rightarrow 0$ is the same as in Theorem 1.1, i.e., exponentially decaying.

In the case of purely discrete spectrum or the case of isolated eigenvalues, we can approximate an eigenfunction also in energy norm by a sequence of finite dimensional eigenvectors: namely, for an isolated eigenvalue $\widetilde{\lambda}$ with normalised eigenfunction $\widetilde{\Phi}$, there is a sequence $\left(\Phi_{m}\right)_{m}$ of normalised functions consisting of a linear combination of eigenfunctions with eigenvalues close to $\widetilde{\lambda}$ such that

$$
\left\|J \Phi_{m}-\widetilde{\Phi}\right\|_{\text {dom } \widetilde{\mathcal{E}}} \leq C_{\widetilde{\lambda}} \delta_{m}
$$

where $C_{\widetilde{\lambda}}$ depends only on $\widetilde{\lambda}$. Here, $\|u\|_{\operatorname{dom} \widetilde{\mathcal{E}}}^{2}=\|u\|_{\mathrm{L}_{2}(K, \mu)}^{2}+\widetilde{\mathcal{E}}(u)$ denotes the energy norm. It follows that the convergence also holds with respect to the original Hilbert space norm of $\widetilde{\mathscr{H}}$. We assume here tacitly that the range of $J$ lies in $\operatorname{dom} \widetilde{\mathcal{E}}$; the more general case is treated in Proposition 2.6.

1.5. Remark. We would like to stress that a result similar to Theorem 1.1 holds also for non-compact spaces such as non-compact fractafolds. A fractafold is a space which is locally homeomorphic to a given pcf self-similar fractal $K$ (see e.g. [Str03]). A non-compact example is given by $X=\bigcup_{m \in \mathbb{N}} F^{-m}(K)$ for some pcf self-similar fractal $K$ with IFS $F$, where $F^{-m}(K)=$ $\bigcup_{w \in W_{m}} F_{w}^{-1}(K)$ for $m \geq 0$ (with the convention that $F_{w}^{-1}$ is the inverse of $F_{w}$ ). We also let $G_{m}$ be the associated $m$-level graph approximation (here, the vertices of $G_{m}$ are included in the vertices of $G_{m+1}$, and each graph $G_{m}$ is infinite). In particular, it follows from the abstract theory of [Pos12, Sec. 4.3] that the entire spectrum and the essential spectrum converge. Moreover, we conclude that isolated eigenvalues and their eigenfunctions converge in the sense of (1.4) (see again Proposition 2.6 for the precise statement).

\subsection{Previous works and further developments}

In [FS92] the method of spectral decimation is described for the first time. Roughly speaking, one can calculate with this method the eigenvalues of generation $m+1$ from generation $m$ by the preimage of some rational function. From the spectral decimation method, one can also conclude the convergence of eigenvalues, cf. [FS92 or Shi96, Sec. 3.1]. This method is restricted to certain fractals, and works only for the self-similar measure. Our method works for any Borel regular probability measure of full support.

Mosco Mos94 developed a notion of convergence of energy forms, nowadays called "Mosco convergence" which is equivalent to strong resolvent convergence. Kuwae and Shioya [KS03, Sec. 2, esp. Sec. 2.5] extended the notion of Mosco convergence to the case of varying Hilbert spaces. Our concept corresponds to a generalisation of norm resolvent convergence, see Remark 2.3 for the relation with homogenisation theory. In [HT15], there is a quite general approach how (separable) Dirichlet forms can be approximated by Dirichlet forms on finite spaces such that the corresponding forms converge in the sense of Mosco. We will relate Mosco convergence and our generalised norm resolvent convergence in a subsequent publication.

In [IPR $\left.{ }^{+} 10\right]$ the resolvent for the Neumann resp. Dirichlet Laplacian is calculated for pcf self-similar fractals $K$. The Neumann Laplacian is the operator associated with $\mathcal{E}_{K}$, while the Dirichlet Laplacian is the operator associated with $\mathcal{E}_{K}^{\mathrm{D}}:=\mathcal{E}_{K} \uparrow_{\left\{u \in \operatorname{dom} \mathcal{E}_{K}|u|_{V_{0}}=0\right\}}$.

Our approach only needs the existence of a sequence of graphs and energy forms converging in a suitable sense to the limit space and limit energy form. In particular, the self-similar 
structure of our fractal is not essential for the proof of Theorem 1.1, and one can extend our results to certain finitely ramified fractals as introduced in Tep08 (see also Remark 4.2).

For numerical calculations of spectra, an adaption of the finite element method (FEM) has been used also for fractals, e.g. in GRS01, ASST03. We comment on this approach in Remark 4.3. Moreover, some of the conditions we have to check for the quasi-unitary equivalence appeared already in the literature in the context of the FEM. For example, let $P_{m} u$ be the piecewise harmonic interpolation spline (using the terminology of Strichartz et al [SU00, GRS01]), i.e., the function with values $u(x)$ for $x \in V_{m}$ and being $m$-harmonic on $K$. Then $P_{m} u=J J^{\prime 1} u$ in our terminology of Subsection 4.1. Moreover, the estimate

$$
\left\|u-P_{m} u\right\|_{L_{2}(K, \mu)}^{2} \leq \frac{r_{0}}{N} \mathcal{E}_{K}(u)
$$

for $u \in \operatorname{dom} \mathcal{E}_{K}$ for symmetric fractals (with common energy renormalisation factor $r_{0} \in(0,1)$ ) appearing in the proof of Theorem 1.1 in Subsection 4.1 is already shown in [GRS01, Thm. 3.4].

We will apply the concept of quasi-unitary equivalence also to the case of "graph-like" spaces such as metric graphs and graph-like manifolds in a second publication [PS18]. We will show that under suitable assumptions, a fractal energy form can be approximated by a suitably renormalised energy form on a family of metric graphs or graph-like spaces. This will complement some numerical results of "outer" approximations resp. approximations of fractals by open domains with their natural Neumann Laplacians as discussed e.g. in [BSU08, pp 50-51, Fig.14-15] and [BHS09].

We are confident that our method also works for approximations of magnetic Laplacians on fractals by discrete magnetic Laplacians as described in [HT13, $\mathrm{HR} 16, \mathrm{HKM}^{+} 17, \mathrm{BCH}^{+} 17$ ], hence allowing to show quasi-unitary equivalence for more general fractals (and not only those where the spectral decimation method is valid).

\subsection{Structure of the article}

In Section 2, we give a brief review of the abstract convergence result for energy forms in different Hilbert spaces. Section 3 contains a brief introduction to pcf fractals in the way we need it. Section 4 - the core of the paper - contains the proofs of our main results. In Section 5 we present some examples.

\subsection{Acknowledgements}

We would like to thank Michael Hinz for helpful comments on the manuscript and literature. We would also like to thank the anonymous referee for carefully reading the manuscript and helpful comments.

\section{An abstract norm resolvent convergence result}

\subsection{Quasi-unitary equivalence}

In this section, we define a sort of "distance" between two operators $\Delta$ and $\widetilde{\Delta}$ defined via energy forms $\mathcal{E}$ and $\widetilde{\mathcal{E}}$, which act in different Hilbert spaces $\mathscr{H}$ and $\mathscr{\mathscr { H }}$, respectively. The general theory is developed in great detail in [Pos12, Ch. 4]. The concept of quasi-unitary equivalence assures a generalised norm resolvent convergence for operators $\Delta=\Delta_{m}$ converging to $\widetilde{\Delta}$ as $m \rightarrow \infty$. Each operator $\Delta_{m}$ acts in a Hilbert space $\mathscr{H}_{m}$ for $m \in \mathbb{N}$; and the Hilbert spaces are allowed to depend on $m$. 
In our application, the Hilbert spaces $\mathscr{H}=\mathscr{H}_{m}=\ell_{2}\left(V_{m}, \mu_{m}\right)$ are functions on the vertices $V_{m}$ of a weighted graph $\left(G_{m}, \mu_{m}\right)$. Moreover, the "limit" metric measure space is $(X, \mu)$ with Hilbert space $\widetilde{\mathscr{H}}=\mathrm{L}_{2}(X, \mu) \cdot 1$

We start now with the general concept:

Let $\mathscr{H}$ and $\widetilde{\mathscr{H}}$ be two separable Hilbert spaces. We say that $\mathcal{E}$ is an energy form in $\mathscr{H}$ if $\mathcal{E}$ is a closed, non-negative quadratic form in $\mathscr{H}$, i.e., if $\mathcal{E}(f):=\mathcal{E}(f, f)$ for some sesquilinear form $\mathcal{E}: \mathscr{H}^{1} \times \mathscr{H}^{1} \longrightarrow \mathbb{C}$, denoted by the same symbol, if $\mathcal{E}(f) \geq 0$ and if $\mathscr{H}^{1}:=\operatorname{dom} \mathcal{E}$, endowed with the norm defined by

$$
\|f\|_{1}^{2}:=\|f\|_{\mathscr{H}}^{2}+\mathcal{E}(f)
$$

is itself a Hilbert space and dense (as a set) in $\mathscr{H}$. We call the corresponding non-negative, self-adjoint operator $\Delta$ (see e.g. [Kat66, Sec. VI.2]) the energy operator associated with $\mathcal{E}$. Similarly, let $\widetilde{\mathcal{E}}$ be an energy form in $\widetilde{\mathscr{H}}$ with energy operator $\widetilde{\Delta}$.

Associated with an energy operator $\Delta$, we introduce a natural scale of Hilbert spaces $\mathscr{H}^{k}$ defined via the abstract Sobolev norms

$$
\|f\|_{k}:=\left\|(\Delta+1)^{k / 2} f\right\| .
$$

Then $\mathscr{H}^{k}=\operatorname{dom} \Delta^{k / 2}$ if $k \geq 0$ and $\mathscr{H}^{k}$ is the completion of $\mathscr{H}=\mathscr{H}^{0}$ with respect to the norm $\|\cdot\|_{k}$ for $k<0$. Obviously, the scale of Hilbert spaces for $k=1$ and its associated norm agrees with $\mathscr{H}^{1}$ and $\|\cdot\|_{1}$ defined above (see [Pos12, Sec. 3.2] for details). Similarly, we denote by $\widetilde{\mathscr{H}^{k}}$ the scale of Hilbert spaces associated with $\widetilde{\Delta}$.

We now need pairs of so-called identification operators acting on the Hilbert spaces and later also pairs of identification operators acting on the form domains.

2.1. Definition. Let $\delta \geq 0$, and let $J: \mathscr{H} \longrightarrow \widetilde{\mathscr{H}}$ and $J^{\prime}: \widetilde{\mathscr{H}} \longrightarrow \mathscr{H}$, resp. $J^{1}: \mathscr{H}^{1} \longrightarrow \widetilde{\mathscr{H}^{1}}$ and $J^{1}: \widetilde{\mathscr{H}^{1}} \longrightarrow \mathscr{H}^{1}$ be bounded linear operators on the Hilbert spaces and energy form domains.

(i) We say that $J$ is $\delta$-quasi-unitary with $\delta$-quasi-adjoint $J^{\prime}$ if

$$
\begin{array}{rrr}
\|J f\| \leq(1+\delta)\|f\|, & \left|\langle J f, u\rangle-\left\langle f, J^{\prime} u\right\rangle\right| \leq \delta\|f\|\|u\| & (f \in \mathscr{H}, u \in \widetilde{\mathscr{H}}), \\
\left\|f-J^{\prime} J f\right\| \leq \delta\|f\|_{1}, & \left\|u-J J^{\prime} u\right\| & \leq \delta\|u\|_{1}
\end{array}
$$

(ii) We say that $J^{1}$ and $J^{\prime 1}$ are $\delta$-compatible with the identification operators $J$ and $J^{\prime}$ if

$$
\left\|J^{1} f-J f\right\| \leq \delta\|f\|_{1}, \quad\left\|J^{\prime 1} u-J^{\prime} u\right\| \leq \delta\|u\|_{1} \quad\left(f \in \mathscr{H}^{1}, u \in \widetilde{\mathscr{H}^{1}}\right) .
$$

(iii) We say that the energy forms $\mathcal{E}$ and $\widetilde{\mathcal{E}}$ are $\delta$-close if

$$
\left|\widetilde{\mathcal{E}}\left(J^{1} f, u\right)-\mathcal{E}\left(f, J^{\prime 1} u\right)\right| \leq \delta\|f\|_{1}\|u\|_{1} \quad\left(f \in \mathscr{H}^{1}, u \in \widetilde{\mathscr{H}}^{1}\right) .
$$

(iv) We say that $\mathcal{E}$ and $\widetilde{\mathcal{E}}$ are $\delta$-quasi-unitarily equivalent (on $\mathscr{H}$ and $\widetilde{\mathscr{H}}$ ), if (2.3a)-(2.3d) are fulfilled, i.e.,

- if there exists identification operators $J$ and $J^{\prime}$ such that $J$ is $\delta$-quasi-unitary with $\delta$-adjoint $J^{\prime}$ (i.e., 2.3a) $2.3 \mathrm{~b}$ hold);

- if there exists identification operators $J^{1}$ and $J^{\prime 1}$ which are $\delta$-compatible with $J$ and $J^{\prime}$ (i.e., 2.3c holds);

- and if $\mathcal{E}$ and $\widetilde{\mathcal{E}}$ are $\delta$-close (i.e., (2.3d) holds).

At first sight the previous definition looks a bit technical, but we will see in Section 4 that it fits perfectly for our approximation of energy forms on fractals by energy forms on graphs and is easy to check. Note that $2.3 \mathrm{~b}$ ) is equivalent with $1.3 \mathrm{a}$ in operator norm notation.

\footnotetext{
${ }^{1}$ It is a question of interpretation, which object is simpler and which one wants to approximate. In the first application of the concept of quasi-unitary equivalence, the space $\mathscr{H}$ was the limit space, the $\mathrm{L}_{2}$-space over a metric graph, while $\widetilde{\mathscr{H}}$ was based on a family of shrinking ("graph-like") manifolds.
} 
2.2. Remark. Let us state some trivial consequences, explaining the notation in two extreme cases:

(i) " $\delta$-quasi-unitary equivalence" is a quantitative generalisation of "unitary equivalence": Note that if $\delta=0, J$ is 0-quasi-unitary if and only if $J$ is unitary with $J^{*}=J^{\prime}$. Moreover, $\mathcal{E}$ and $\widetilde{\mathcal{E}}$ are 0-quasi-unitarily equivalent if and only if $\Delta$ and $\widetilde{\Delta}$ are unitarily equivalent (in the sense that $J R=\widetilde{R} J$ ).

(ii) " $\delta_{m}$-quasi-unitary equivalence" with $\delta_{m} \rightarrow 0$ is a generalisation of " $n$ orm resolvent convergence": If $\mathscr{H}=\widetilde{\mathscr{H}}, J=J^{\prime}=\mathrm{id}_{\mathscr{H}}$, then the first two conditions (2.3a) $-(2.3 \mathrm{~b})$ are trivially fulfilled with $\delta=0$. Moreover, if $\Delta=\Delta_{m}$ and $\delta=\delta_{m} \rightarrow 0$ as $m \rightarrow \infty$, then $\widetilde{\mathcal{E}}$ and $\mathcal{E}_{m}$ are $\delta_{m}$-quasi-unitarily equivalent if and only if $\left\|R_{m}^{-1 / 2}\left(R_{m}-\widetilde{R}\right) \widetilde{R}^{-1 / 2}\right\| \rightarrow 0$ as $m \rightarrow \infty$, and hence it follows that $\left\|R_{m}-\widetilde{R}\right\| \rightarrow 0$, i.e., $\Delta_{m}$ converges to $\widetilde{\Delta}$ in norm resolvent sense as $m \rightarrow \infty$.

2.3. Remark. If $\mathcal{E}_{m}$ and $\widetilde{\mathcal{E}}$ are $\delta_{m}$-quasi-unitarily equivalent, then the corresponding operators also converge in the sense of Kuwae and Shioya [KS03, Sec. 2.5]. Our concept is a generalisation of norm resolvent convergence while Kuwae and Shioya provide a generalisation of strong resolvent convergence, i.e., convergence of the resolvents in the pointwise operator topology to the case of varying Hilbert spaces. Their convergence is equivalent to a version of Mosco convergence of forms in varying spaces, see [KS03, Thm. 2.4]. We discuss such questions in a subsequent paper.

Similarly, in homogenisation problems, usually strong resolvent convergence is shown, and hence a typical assumption is compactness of the resolvent (i.e., compactness of the domain). In [KP18] the first author and Khrabustovskyi show that in the situation of the Dirichlet Laplacian on a domain with many small periodic obstacles removed one has $\delta_{\varepsilon}$-quasi-unitary equivalence with a homogenised operator, where $\varepsilon$ is the order of periodicity. Apart from [DCR18] this is the first time (to the best of our knowledge) where norm resolvent convergence is shown directly for homogenisation problems.

Moreover, although the concept of quasi-unitary equivalence is stronger than e.g. Mosco convergence, it seems to be not very difficult to show the conditions in Definition 2.1 in applications such at graphs approximating self-similar fractals or in the above homogenisation setting of [KP18].

Note that the conditions in Definition 2.1 are not written in stone. It will be convenient in our case to use the following modification:

2.4. Lemma. Assume that 2.3a is fulfilled with $\delta_{\mathrm{a}}>0$ and (2.3c) with $\delta_{\mathrm{c}}>0$. If

$$
\left\|u-J J^{\prime 1} u\right\| \leq \delta^{\prime}\|u\|_{1} \quad\left(u \in \widetilde{\mathscr{H}^{1}}\right)
$$

holds, then the second inequality in $(2.3 \mathrm{~b})$ is fulfilled with $\delta=\delta^{\prime}+\left(1+\delta_{\mathrm{a}}\right) \delta_{\mathrm{c}}$.

In particular, if all conditions (2.3) are fulfilled for some $\delta>0$, except for the second one in 2.3b which is replaced by (2.4), then $\mathcal{E}$ and $\widetilde{\mathcal{E}}$ are $\widetilde{\delta}$-quasi-unitarily equivalent with $\widetilde{\delta}=\delta^{\prime}+(1+\delta) \delta$.

Proof. We have

$$
\begin{aligned}
\left\|u-J J^{\prime} u\right\| & \leq\left\|u-J J^{\prime 1} u\right\|+\left\|J\left(J^{1}-J^{\prime}\right) u\right\| \\
& \leq\left\|u-J J^{\prime 1} u\right\|+\|J\|\left\|\left(J^{\prime 1}-J^{\prime}\right) u\right\| \leq\left(\delta^{\prime}+\left(1+\delta_{\mathrm{a}}\right) \delta_{\mathrm{c}}\right)\|u\|_{1}
\end{aligned}
$$

and if $\delta^{\prime}, \delta_{\mathrm{a}}, \delta_{\mathrm{c}} \leq \delta$, then the error estimate is greater or equal to $2 \delta+\delta^{2}$, as claimed.

\subsection{Consequences of quasi-unitary equivalence}

Let us mention some consequences of the above-mentioned quasi-unitary equivalence we need in the proof of Proposition 2.6. 
We first provide the notation $\|A\|_{-1 \rightarrow 1}=\left\|(\widetilde{\Delta}+1)^{1 / 2} A(\Delta+1)^{1 / 2}\right\|$ for $A: \mathscr{H}^{-1} \longrightarrow \widetilde{\mathscr{H}^{1}}$. Moreover, $\left(J^{1}\right)^{*}: \mathscr{H}^{-1} \longrightarrow \widetilde{\mathscr{H}}^{-1}$ denotes the dual map of $J^{1}: \widetilde{\mathscr{H}}^{1} \longrightarrow \mathscr{H}^{1}$ with respect to the dual pairing $\mathscr{H}^{1} \times \mathscr{H}^{-1}$ induced by the inner product on $\mathscr{H}$ and similarly on $\widetilde{\mathscr{H}}$.

2.5. Proposition (see [Pos12, Ch. 4]). Let $\eta:[0, \infty) \longrightarrow \mathbb{C}$ be a function continuous in a neighbourhood $U$ of $\sigma(\widetilde{\Delta})$ such that $\lim _{\lambda \rightarrow \infty}(\lambda+1)^{1 / 2} \eta(\lambda)$ exists. Then there is a function $\delta \mapsto \varepsilon(\delta)$ such that $\varepsilon(\delta) \rightarrow 0$ if $\delta \rightarrow 0$ and

$$
\begin{aligned}
\left\|J^{\prime} \eta(\widetilde{\Delta})-\eta(\Delta) J^{\prime}\right\| & \leq \varepsilon(\delta), \\
\left|\left\|J^{\prime} u\right\|-\|u\|\right| & \leq 3 \delta\|u\|_{1} \\
\left\|J^{1} \eta(\Delta)-\eta(\widetilde{\Delta})\left(J^{\prime 1}\right)^{*}\right\|_{-1 \rightarrow 1} & \leq \varepsilon(\delta)
\end{aligned}
$$

for any pair of $\delta$-quasi-unitarily equivalent energy forms $\mathcal{E}$ and $\widetilde{\mathcal{E}}$ with associated operators $\Delta$ and $\widetilde{\Delta}$, where $\varepsilon=\varepsilon_{\eta}$ depends only on $\eta$ and $U$. If $\eta(\lambda)=\mathrm{e}^{-t \lambda}$ (for $t>0$ ) or $\eta=\mathbb{1}_{D}$ (if $\partial D \cap \sigma(\widetilde{\Delta})=\emptyset$ for an open set $D \subset \mathbb{C}$ with smooth boundary) we can choose $\varepsilon(\delta)=C \delta$ for some constant $C>0$.

An inequality similar to $2.5 \mathrm{~b}$ ) is proven in 2.10 in a more specific situation.

2.6. Proposition. Let $\mathcal{E}$ and $\widetilde{\mathcal{E}}$ be two $\delta$-quasi-unitarily equivalent energy forms with associated operators $\Delta$ and $\widetilde{\Delta}$. Assume that $\widetilde{\Phi}$ is a normalised eigenvector of $\widetilde{\Delta}$, such that its eigenvalue $\widetilde{\lambda}$ is discrete in $\sigma(\widetilde{\Delta})$, i.e., there is an open disc $D$ in $\mathbb{C}$ such that $\sigma(\widetilde{\Delta}) \cap D=\{\widetilde{\lambda}\}$. Then there exists a normalised eigenvector $\Phi$ of $\Delta$ with $\Phi \in \operatorname{ran} \mathbb{1}_{D}(\Delta)$ and a universal constant $C$ depending only on $\tilde{\lambda}$ (and the radius of $D$ ) such that

$$
\left\|J^{1} \Phi-\widetilde{\Phi}\right\|_{1} \leq C \delta
$$

for $\delta>0$ small enough.

Note that the eigenvalue $\widetilde{\lambda}$ does not necessarily need to have finite multiplicity.

Proof. Set $P:=\mathbb{1}_{D}(\Delta)$ and $\widetilde{P}:=\mathbb{1}_{D}(\widetilde{\Delta})$. Note that ran $P$ may consist of the linear combination of several eigenvectors if $\tilde{\lambda}$ is not a simple eigenvalue. We have

$$
\left\|P J^{\prime} \widetilde{\Phi}\right\| \geq\left\|J^{\prime} \widetilde{P} \widetilde{\Phi}\right\|-\left\|\left(P J^{\prime}-J^{\prime} \widetilde{P}\right) \widetilde{\Phi}\right\| \geq\left\|J^{\prime} \widetilde{\Phi}\right\|-C_{\eta} \delta\|\widetilde{\Phi}\| \geq 1-\underbrace{\left(3(\widetilde{\lambda}+1)^{1 / 2}+C_{\eta}\right)}_{=: C_{1}} \delta
$$

by 2.5a with $\eta=\mathbb{1}_{D}$ and $2.5 \mathrm{~b}$ and similarly,

$$
\left\|P J^{\prime} \widetilde{\Phi}\right\| \leq\left\|J^{\prime} \widetilde{P} \widetilde{\Phi}\right\|+\left\|\left(P J^{\prime}-J^{\prime} \widetilde{P}\right) \widetilde{\Phi}\right\| \leq\left\|J^{\prime} \widetilde{\Phi}\right\|+C_{\eta} \delta\|\widetilde{\Phi}\| \leq 1+C_{1} \delta
$$

and therefore

$$
\left|\left\|P J^{\prime} \widetilde{\Phi}\right\|-1\right| \leq C_{1} \delta
$$

In particular, $\left\|P J^{\prime} \widetilde{\Phi}\right\|>0$ for $\delta$ small enough. Let

$$
\Phi:=\frac{1}{\left\|P J^{\prime} \widetilde{\Phi}\right\|} P J^{\prime} \widetilde{\Phi}
$$


then $\Phi \in \mathscr{H}^{1}$ and for $\delta<1 / C_{1}$ we have

$$
\begin{aligned}
\left\|J^{1} \Phi-\widetilde{\Phi}\right\|_{1} & =\left\|\frac{1}{\left\|P J^{\prime} \widetilde{\Phi}\right\|} J^{1} P J^{\prime} \widetilde{\Phi}-\widetilde{\Phi}\right\|_{1} \\
& \leq \frac{1}{\left\|P J^{\prime} \widetilde{\Phi}\right\|}\left(\left\|\left(J^{1} P-\widetilde{P}\left(J^{\prime 1}\right)^{*}\right) J^{\prime} \widetilde{\Phi}\right\|_{1}+\left\|\widetilde{P}\left(\left(J^{\prime 1}\right)^{*} J^{\prime} \widetilde{\Phi}-\widetilde{\Phi}\right)\right\|_{1}\right. \\
& \left.\quad+\left|1-\left\|P J^{\prime} \widetilde{\Phi}\right\|\right|\|\widetilde{\Phi}\|_{1}\right) \\
\leq & \frac{1}{1-C_{1} \delta}\left(C_{\eta} \delta\|\widetilde{\Phi}\|_{-1}+(\widetilde{\lambda}+1)\left\|\left(J^{\prime 1}\right)^{*} J^{\prime}-\mathrm{id}\right\|_{0 \rightarrow-1}\|\widetilde{\Phi}\|_{1}+C_{1} \delta\|\widetilde{\Phi}\|_{1}\right) \\
& \leq \frac{1}{1-C_{1} \delta}\left(C_{\eta}+(\widetilde{\lambda}+1)^{3 / 2} C^{\prime \prime}+C_{1}(\widetilde{\lambda}+1)^{1 / 2}\right) \delta
\end{aligned}
$$

using 2.5c), $\|\widetilde{P}\|_{-1 \rightarrow 1}=1+\widetilde{\lambda}$ and $(2.6)$ for the second inequality, and

$$
\begin{aligned}
\left\|\left(J^{\prime 1}\right)^{*} J^{\prime}-\mathrm{id}\right\|_{0 \rightarrow-1}=\left\|\left(J^{\prime}\right)^{*} J^{\prime 1}-\mathrm{id}\right\|_{1 \rightarrow 0} & \leq\left\|\left(J^{\prime}\right)^{*}-J\right\|\left\|J^{\prime 1}\right\|_{1 \rightarrow 0}+\left\|J J^{\prime 1}-\mathrm{id}\right\|_{1 \rightarrow 0} \\
& \leq \delta(1+3 \delta)+\delta^{\prime} \leq\left(1+3 / C_{1}+C^{\prime}\right) \delta=: C^{\prime \prime} \delta
\end{aligned}
$$

using also

$$
\left\|J^{\prime 1}\right\|_{1 \rightarrow 0} \leq\left\|J^{\prime 1}-J^{\prime}\right\|_{1 \rightarrow 0}+\left\|J^{\prime}-J^{*}\right\|_{0 \rightarrow 0}+\left\|J^{*}\right\|_{0 \rightarrow 0} \leq 1+3 \delta \leq 1+3 / C_{1}
$$

as $\|A\|_{1 \rightarrow 0} \leq\|A\|_{0 \rightarrow 0}$ and $\delta<1 / C_{1}$, and using $\delta^{\prime} \leq C^{\prime} \delta$ for some constant $C^{\prime}>0$; the latter estimate can be seen similarly as in Lemma 2.4 .

\subsection{Some eigenvalue estimates}

For problems with purely discrete spectrum, we also cite some results dealing directly with eigenvalue estimates, using the min-max principle (see [Pos12, Prp. 4.4.18] for the most general version): Here, $\lambda_{k}$ denotes the $k$-th eigenvalue of the operator associated with $\mathcal{E}$, in increasing order and repeated according to their multiplicity.

2.7. Proposition. Assume that we have a first order identification operator

$$
J^{1}: \mathscr{H}^{1} \longrightarrow \widetilde{\mathscr{H}^{1}}
$$

such that there exist $\delta_{0} \geq 0$ and $\delta_{1} \geq 0$ with

$$
\left\|J^{1} f\right\|^{2} \geq\|f\|^{2}-\delta_{0}\|f\|^{2}-\delta_{1} \mathcal{E}(f) \quad \text { and } \quad \widetilde{\mathcal{E}}\left(J^{1} f\right) \leq \mathcal{E}(f)
$$

for all $f \in \mathscr{H}^{1}$. If $\delta_{0}+\delta_{1} \lambda_{k}<1$ then

$$
\widetilde{\lambda}_{k} \leq \frac{1}{1-\delta_{0}-\delta_{1} \lambda_{k}} \cdot \lambda_{k} \quad \text { or } \quad \frac{1-\delta_{0}}{1+\delta_{1} \widetilde{\lambda}_{k}} \cdot \widetilde{\lambda}_{k} \leq \lambda_{k}
$$

for all $k \in \mathbb{N}$.

If we have shown quasi-unitary equivalence and one additional assumption, then we can conclude from the last proposition (again slightly adopted to our application in Section 4 in order to get an optimal estimate):

2.8. Proposition. Assume that $\mathcal{E}$ and $\widetilde{\mathcal{E}}$ are $\delta$-quasi-unitarily equivalent (with $2.3 \mathrm{a})$ and $(2.3 \mathrm{~d})$ fulfilled with $\delta=0$ and (2.3b) and (2.3c) with $\|f\|_{1}$ resp. $\|u\|_{1}$ replaced by $\mathcal{E}(f)^{1 / 2}$ resp. $\left.\widetilde{\mathcal{E}}(u)^{1 / 2}\right)$ and that

$$
\mathcal{E}\left(f, J^{\prime 1} J^{1} f-f\right) \leq 0
$$

for all $f \in \mathscr{H}^{1}$, then $(2.8)$ is fulfilled with $\delta_{0}=\delta_{1}=\delta$. Moreover, if

$$
\widetilde{\mathcal{E}}\left(u, J^{1} J^{\prime 1} u-u\right) \leq 0
$$

for all $u \in \widetilde{\mathscr{H}^{1}}$, then

$$
\lambda_{k} \leq \frac{1}{1-\delta\left(1+\widetilde{\lambda}_{k}\right)} \cdot \widetilde{\lambda}_{k} \quad \text { or } \quad \frac{1-\delta}{1+\delta \lambda_{k}} \cdot \lambda_{k} \leq \widetilde{\lambda}_{k}
$$


Proof. We first estimate

$$
|\|J f\|-\|f\||=\frac{\left|\|J f\|^{2}-\|f\|^{2}\right|}{\|J f\|+\|f\|}=\frac{\left|\left\langle J^{*} J f-f, f\right\rangle\right|}{\|J f\|+\|f\|} \stackrel{\operatorname{CS}}{\leq} \frac{\delta \mathcal{E}(f)^{1 / 2}\|f\|}{\|f\|}=\delta \mathcal{E}(f)^{1 / 2}
$$

for $f \in \mathscr{H}^{1}$ using $\left.2.3 \mathrm{~b}\right)$; this implies

$$
\left\|J^{1} f\right\|-\|f\| \geq(\|J f\|-\|f\|)-\left\|\left(J-J^{1}\right) f\right\| \geq-2 \delta \mathcal{E}(f)^{1 / 2}
$$

using the previous estimate and $(2.3 \mathrm{c})$. We conclude that

$$
\left\|J^{1} f\right\|^{2}-\|f\|^{2}=\left(\left\|J^{1} f\right\|-\|f\|\right)\left(\left\|J^{1} f\right\|+\|f\|\right) \geq-2 \delta \mathcal{E}(f)^{1 / 2}\|f\| \stackrel{\mathrm{CY}}{\geq}-\delta\left(\|f\|^{2}+\mathcal{E}(f)\right) .
$$

For the energy forms, we have

$$
\widetilde{\mathcal{E}}\left(J^{1} f\right)-\mathcal{E}(f)=\left(\widetilde{\mathcal{E}}\left(J^{1} f, J^{1} f\right)-\mathcal{E}\left(f, J^{1} J^{1} f\right)\right)+\mathcal{E}\left(f, J^{\prime 1} J^{1} f-f\right) \leq 0
$$

using 2.3d) (with $\delta=0$ ) and 2.9a). The result follows from Proposition 2.7.

The second estimate can be seen similarly by swapping $\mathcal{E}$ and $\widetilde{\mathcal{E}}$, using the identification operators in the opposite direction and $2.9 \mathrm{~b}$

\section{Post-critically finite fractals and self-similar energy forms}

In this section, we briefly review some facts on fractals and self-similar energy forms, see [Kig01] or [Str06, Ch. 4]. Let $(X, d)$ be a complete metric space. Typically, we work with $X=\mathbb{R}^{d}$ with the Euclidean metric (see Section 5), but our abstract convergence results also hold in the general case.

\subsection{Post-critically finite self-similar fractals and their approximating graphs}

We start with a finite family $F=\left(F_{j}\right)_{j=1, \ldots, N}$ of contractive similarities $F_{j}: X \longrightarrow X$, i.e.,

$$
d\left(F_{j}(x), F_{j}(y)\right)=\theta_{j} d(x, y)
$$

for all $x, y \in X$, where $\theta_{j} \in(0,1)$ denotes the contraction ratio. By the Banach fixed point theorem, such a similarity has a unique fixed point $q_{j} \in X$. The family $F$ is called an iterated function system (IFS). For any IFS, there exists a unique (non-empty) compact subset $K \subset X$, called self-similar set or self-similar fractal, such that $K$ is self-similar with respect to $F$, i.e., such that (1.1) holds. The existence is guaranteed by Banach's fixed point theorem on the space of compact subsets of $X$ with Hausdorff distance (see [Kig01, Thm. 1.1.7]).

For a word $w=w_{1} \ldots w_{m}$ of length $|w|=m$ over the alphabet $\{1, \ldots, N\}$ we define the $\operatorname{map} F_{w}$ by $F_{w}=F_{w_{1}} \circ \cdots \circ F_{w_{m}}$. We denote by $W_{m}=\{1, \ldots, N\}^{m}$ the set of all words of length $m$. Then there is a natural cell structure on the self-similar set $K$ described by the map $W_{m} \ni w \mapsto F_{w}(K)$ and we call $K_{w}:=F_{w}(K)$ an $m$-cell.

3.1. Definition. We say that a self-similar set $K$ is post-critically finite (or pcffor short) if $K$ is connected, and if there exists a finite set $V_{0} \subset K$, called the boundary of $K$, such that

$$
F_{w}(K) \cap F_{w^{\prime}}(K) \subset F_{w}\left(V_{0}\right) \cap F_{w^{\prime}}\left(V_{0}\right)
$$

for all words $w, w^{\prime}\left(w \neq w^{\prime}\right)$ of the same length. Moreover, we assume that each boundary point is a fixed point of the IFS, i.e., that $V_{0} \subset\left\{q_{1}, \ldots, q_{N}\right\}$.

In other words, on a pcf self-similar fractal, its $m$-cells intersect only in the corresponding $m$-cell boundary points, hence in a finite set of at most $N$ elements.

For $m=0$, we let $G_{0}=\left(V_{0}, E_{0}\right)$ be the complete graph. For $m \in \mathbb{N}$, we denote by

$$
V_{m}:=\bigcup_{w \in W_{m}} F_{w}\left(V_{0}\right)
$$


the union of all $m$-cell boundary points and $V_{0}$. We consider $V_{m}$ as the vertex set of a graph $G_{m}$ with edges

$$
E_{m}:=\left\{\{x, y\} \subset V_{m} \mid x \neq y \text { and there is } w \in W_{m} \text { such that } x, y \in F_{w}\left(V_{0}\right)\right\} .
$$

We also write $x \sim_{m} y$ if $\{x, y\} \in E_{m}$. We call $x \in V_{m}$ a junction point if $x$ lies in more than one $m$-cell. It is clear that once, the IFS and the graph $G_{0}=\left(V_{0}, E_{0}\right)$ at level $m=0$ are given, the sequence of graphs $G_{m}=\left(V_{m}, E_{m}\right)$ is recursively defined. We call $\left(G_{m}\right)_{m \in \mathbb{N}_{0}}$ the approximating graph sequence associated with the self-similar set $K$ and its boundary $V_{0}$.

\subsection{Energy forms on the approximating graphs}

Associated with the graph $G_{m}$ is its energy, given by the quadratic form

$$
\mathcal{E}_{G_{m}}(f)=\sum_{\{x, y\} \in E_{m}} c_{\{x, y\}, m}|f(x)-f(y)|^{2}
$$

for functions $f \in \ell\left(V_{m}\right):=\left\{f: V_{m} \longrightarrow \mathbb{C}\right\}$; we specify the conductances $c_{\{x, y\}, m}>0$ whenever $x \sim_{m} y$ later on.

3.2. Definition ([Kig01, Def. 2.2.1]). We say that a sequence $\left(\mathcal{E}_{G_{m}}\right)_{m \in \mathbb{N}_{0}}$ of energy forms on a sequence of graphs $\left(G_{m}\right)_{m \in \mathbb{N}_{0}}$ is compatible, if the vertex sets of $G_{m}$ are nested (i.e., $V_{m} \subset V_{m+1}$ ) and if

$$
\mathcal{E}_{G_{m}}(\varphi)=\min \left\{\mathcal{E}_{G_{m+1}}(f) \mid f \in \ell\left(V_{m+1}\right), f \uparrow_{V_{m}}=\varphi\right\}
$$

for all $\varphi \in \ell\left(V_{m}\right)$. The (unique) minimiser $h_{m+1} \in \ell\left(V_{m+1}\right)$ of $(3.2)$ is called harmonic extension of $\varphi \in \ell\left(V_{m}\right)$.

Remark. The right hand side of $(3.2)$ can be seen as a Dirichlet-to-Neumann form of the graph $V_{m+1}$ with boundary $V_{m}$; compatibility then means that the $(m+1)$-th Dirichlet-to-Neumann form actually agrees with the $m$-th graph energy $\mathcal{E}_{G_{m}}$ (see e.g. [Kig01, Sec. 2.1] or [Pos16, Sec. 6.7] for this interpretation).

For a compatible sequence $\left(\mathcal{E}_{G_{m}}\right)_{m}$ we can define a limit form (see [Kig01, Sec. 2.2]). Let $V_{*}:=\bigcup_{m \in \mathbb{N}_{0}} V_{m}$ and $u \in \ell\left(V_{*}\right)$. As $u \uparrow_{V_{m+1}}$ is an extension of $u \uparrow_{V_{m}}$, we have

$$
\mathcal{E}_{G_{m}}\left(u \uparrow_{V_{m}}\right) \leq \mathcal{E}_{G_{m+1}}\left(u \uparrow_{V_{m+1}}\right)
$$

by 3.2 , hence

$$
\mathcal{E}_{K}(u):=\lim _{m \rightarrow \infty} \mathcal{E}_{G_{m}}\left(u \uparrow_{V_{m}}\right)
$$

exists (and may be $\infty$ ).

3.3. Definition. Let $\left(G_{m}\right)_{m \in \mathbb{N}_{0}}$ be the approximating sequence of graphs associated with the IFS $\left(F_{j}\right)_{j=1, \ldots, N}$, the corresponding self-similar set $K$ and its boundary $V_{0}$. We call a sequence $\left(\mathcal{E}_{G_{m}}\right)_{m \in \mathbb{N}_{0}}$ of energy forms on $\left(G_{m}\right)_{m}$ self-similar if there exist so-called renormalisation factors $r_{j} \in(0,1)$ for $j=1, \ldots, N$ such that

$$
\mathcal{E}_{G_{m+1}}(u)=\sum_{j=1}^{N} r_{j}^{-1} \mathcal{E}_{G_{m}}\left(u \circ F_{j}\right)
$$

for $u \in \ell\left(V_{m+1}\right)$.

Clearly, a self-similar sequence $\left(\mathcal{E}_{G_{m}}\right)_{m}$ can be defined recursively, given $\mathcal{E}_{G_{0}}$ on $\ell\left(V_{0}\right)$ by (3.4). As a result, we then obtain

$$
\mathcal{E}_{G_{m}}(u)=\sum_{w \in W_{m}} r_{w}^{-1} \mathcal{E}_{G_{0}}\left(u \circ F_{w}\right)
$$

where $r_{w}:=r_{w_{1}} \cdot \ldots \cdot r_{w_{m}}$. In particular, the conductances are given by

$$
c_{e, m}=\frac{c_{e_{0}, 0}}{r_{w}} \in\left[c_{-, m}, c_{+, m}\right] \quad \text { with } \quad c_{ \pm, m}:=\frac{c_{ \pm, 0}}{\left(r_{\mp}\right)^{m}},
$$


where $w \in W_{m}$ is given by $e=F_{w}\left(e_{0}\right)$. Moreover, $r_{ \pm}$is the maximal resp. minimal value of the renormalisation factors $r_{1}, \ldots, r_{N}$, and similarly $c_{ \pm, 0}$ is the maximal resp. minimal value of the conductances $c_{e_{0}, 0}$ of $\mathcal{E}_{G_{0}}$.

We now make our main assumption on the fractal:

3.4. Definition. We say that a fractal $K$ is approximable by finite weighted graphs, if $K$ is a pcf self-similar set given by an IFS, if there is an approximating sequence of finite weighted graphs $\left(G_{m}\right)_{m \in \mathbb{N}_{0}}$, and if there is a compatible and self-similar sequence $\left(\mathcal{E}_{G_{m}}\right)_{m}$ of energy forms $\mathcal{E}_{G_{m}}$ on $G_{m}$. We call $V_{0}$ the boundary of $K$.

On a fractal $K$ approximable by finite weighted graphs, there exists a natural energy form $\mathcal{E}_{K}$ defined by $(3.3)$. Note that such an energy form is uniquely determined by the data $\left(\left(F_{j}\right)_{j=1, \ldots, N},\left(r_{j}\right)_{j=1, \ldots, N}, \mathcal{E}_{G_{0}}\right)$.

The pcf property guarantees the existence of the approximating sequence of graphs. Moreover, given an energy form $\mathcal{E}_{G_{0}}$ and renormalisation factors $r_{1}, \ldots, r_{N}$, one can define a selfsimilar sequence of energy forms $\left(\mathcal{E}_{G_{m}}\right)_{m}$ by $(3.5)$.

3.5. Remark. The difficult problem is to find $\mathcal{E}_{G_{0}}$ (i.e., conductances $\left.\left(c_{e_{0}, 0}\right)_{e_{0} \in E_{0}}\right)$ and $r_{1}, \ldots, r_{N}$ such that $\left(\mathcal{E}_{G_{m}}\right)_{m}$ (defined via (3.5) and hence self-similar) is at the same time compatible. This problem is called the renormalisation problem, and can be rephrased as a fixed point problem or a non-linear eigenvalue problem. It can be shown (see e.g. [Kig01, Prop. 3.1.3]) that $\left(\mathcal{E}_{G_{m}}\right)_{m}$ defined via (3.5) is compatible if and only if it is compatible at level $m=0$ (i.e., (3.2) holds for $m=0$ ). The renormalisation problem is not (yet) solved for general pcf fractals (see e.g. Str06, Sec.4.2, p. 98f]), but there are many examples (see Section 5).

\subsection{The energy form on the fractal}

Given now a fractal $K$ approximable by finite weighted graphs, we can define a limit form $\mathcal{E}_{K}$ as in (3.3) and the self-similarity (3.4) survives the limit: $\mathcal{E}_{K}$ is self-similar, i.e.,

$$
\mathcal{E}_{K}(u)=\sum_{j=1}^{N} r_{j}^{-1} \mathcal{E}_{K}\left(u \circ F_{j}\right)
$$

holds for all $u \in \ell\left(V_{*}\right)$. Let now $\mathcal{E}_{K}$ be the form defined in 3.3 on

$$
\operatorname{dom} \mathcal{E}_{K}:=\left\{u \in \mathrm{C}(K) \mid \mathcal{E}_{K}(u):=\lim _{m \rightarrow \infty} \mathcal{E}_{G_{m}}\left(u \uparrow_{V_{m}}\right)<\infty\right\} .
$$

As $V_{*}=\bigcup_{m} V_{m}$ is dense in $K$ and as a continuous function on the compact set $K$ is uniformly continuous, $u$ is indeed determined by its values on $V_{*}$.

The compatibility of the sequence $\left(\mathcal{E}_{G_{m}}\right)_{m}$ now passes over to the limit in the following sense (see [Kig01, Lem. 2.2.2]): for any "boundary value" $\varphi \in \ell\left(V_{m}\right)$ there exists a unique continuous function $h \in \operatorname{dom} \mathcal{E}_{K}$ on $K$ such that $h \uparrow_{V_{m}}=\varphi$ and

$$
\mathcal{E}_{G_{m}}(\varphi)=\mathcal{E}_{K}(h)=\min \left\{\mathcal{E}_{K}(u) \mid u \in \operatorname{dom} \mathcal{E}_{K}, u \uparrow_{V_{m}}=\varphi\right\} .
$$

These functions are called $m$-harmonic functions. In the special case where $\varphi$ is the characteristic function $\mathbb{1}_{x}$ of the set $\{x\}$ for $x \in V_{m}$ we denote the $m$-harmonic function with boundary value $\mathbb{1}_{x}$ by $\psi_{x, m}$. By polarisation and a simple argument, it follows for the corresponding sesquilinear forms that

$$
\mathcal{E}_{K}(h, u)=\mathcal{E}_{G_{m}}\left(h \uparrow_{V_{m}}, u \Upsilon_{V_{m}}\right)
$$

for all $u \in \operatorname{dom} \mathcal{E}_{K}$ and $h \in \operatorname{dom} \mathcal{E}_{K}$ an $m$-harmonic function.

From (3.7) we derive the following "localisation" of the energy form $\mathcal{E}_{K}$. For any $w \in W_{m}$ we define $\mathcal{E}_{K_{w}}$ with $\operatorname{dom} \mathcal{E}_{K_{w}}=\left\{u \uparrow_{K_{w}} \mid u \in \operatorname{dom} \mathcal{E}_{K}\right\}$ by

$$
\mathcal{E}_{K_{w}}\left(u \uparrow_{K_{w}}\right):=r_{w}^{-1} \mathcal{E}_{K}\left(u \uparrow_{K_{w}} \circ F_{w}\right)
$$


for all $u \in \operatorname{dom} \mathcal{E}_{K}$. Then we have the following useful "localisation" formula

$$
\mathcal{E}_{K}(u)=\sum_{w \in W_{m}} \mathcal{E}_{K_{w}}\left(u \uparrow_{K_{w}}\right)
$$

for all $u \in \operatorname{dom} \mathcal{E}_{K}$.

As each cell $K_{w}$ described by the IFS $\left\{F_{w j}\right\}_{j=1, \ldots, N}$ is itself a pcf self-similar set, we conclude from the definition of the resistance metric (see [Kig01, Secs. 2.3] or [Str06, Sec. 4.4 and 1.6]): 3.6. Proposition. Let $K$ be a fractal approximable by finite weighted graphs with self-similar energy form $\mathcal{E}_{K}$ and let $x, y \in K_{w}$ be in a cell $K_{w}$ of generation $m$. Then

$$
|u(x)-u(y)|^{2} \leq \frac{r_{w}}{c_{-, 0}} \mathcal{E}_{K_{w}}\left(u \Upsilon_{K_{w}}\right) \leq \frac{1}{c_{-, m}} \mathcal{E}_{K_{w}}\left(u \Upsilon_{K_{w}}\right)
$$

for all $u \in \operatorname{dom} \mathcal{E}_{K}$.

Remark. Note that the localisation of $\mathcal{E}_{K}$ in (3.11) allows us to consider only the energy on $K_{w}$ instead of $K$ on the right hand side. This slight detail will help us to obtain the optimal estimate in the second estimates of $(2.3 \mathrm{~b})$ and $(2.3 \mathrm{c})$ in the proof of Theorem 1.1 in Subsection 4.1.

\subsection{Measures on fractals and graphs and the associated operators}

In order to have a closed form $\mathcal{E}_{K}$ and in order to define an associated self-adjoint operator, we need a Hilbert space structure on $K$, i.e., a measure $\mu$ on $K$. We assume that $\mu$ is a Borel regular probability measure of full support (see e.g. [Kig01, Sec. 1.4]). Examples such as a self-similar measure are given in Subsection 5.1. The Hilbert space is then $\mathrm{L}_{2}(K, \mu)$ with its usual inner product.

The domain dom $\mathcal{E}_{K}$ of $\mathcal{E}_{K}$ is itself a Hilbert space, and it can be shown that the embedding $\operatorname{dom} \mathcal{E}_{K} \subset \mathrm{L}_{2}(K, \mu)$ is compact. This implies that the operator $\Delta_{(K, \mu)}$ associated with the quadratic form $\mathcal{E}_{K}$, i.e., the operator defined via

$$
\mathcal{E}_{K}(u, v)=\left\langle\Delta_{(K, \mu)} u, v\right\rangle_{\mathrm{L}_{2}(K, \mu)}
$$

for all $v \in \operatorname{dom} \mathcal{E}_{K}$, has compact resolvent, hence purely discrete spectrum (note that - in contrast to some literature like Kigami's or Strichartz's books [Kig01, Str06] - our operator is non-negative, i.e, $\left.\Delta_{(K, \mu)} \geq 0\right)$. Moreover, it can be shown that dom $\mathcal{E}_{K} \subset \mathrm{C}(K)$, and it makes sense to evaluate $u \in \operatorname{dom} \mathcal{E}_{K}$ at points of $K$.

For the Hilbert space structure on the graphs $G_{m}$, we also need a measure $\mu_{m}$ on $V_{m}$. We set

$$
\mu_{m}(x)=\int_{K} \psi_{x, m} \mathrm{~d} \mu
$$

and we call the sequence $\left(\mu_{m}\right)_{m}$ approximating measures corresponding to $(K, \mu)$. The measures $\mu_{m}$ are actually discrete probability measures of full support because the family of $m$ harmonic functions $\left\{\psi_{x, m}\right\}_{x \in V_{m}}$ forms a partition of unity and $\mu$ is a probability measure of full support on $K$.

By $\ell_{2}\left(V_{m}, \mu_{m}\right)$ we denote the finite dimensional Hilbert space $\ell\left(V_{m}\right)$ with norm (and hence inner product) given by

$$
\|f\|_{\ell_{2}\left(V_{m}, \mu_{m}\right)}^{2}:=\sum_{x \in V_{m}} \mu_{m}(x)|f(x)|^{2} .
$$

A simple calculation shows that the (bounded) operator $\Delta_{\left(G_{m}, \mu_{m}\right)} \geq 0$ associated with the energy form $\mathcal{E}_{G_{m}}$ acts as

$$
\left(\Delta_{\left(G_{m}, \mu_{m}\right)} f\right)(x)=\frac{1}{\mu_{m}(x)} \sum_{y \sim m x} c_{\{x, y\}, m}(f(x)-f(y)) .
$$




\section{Proof of the quasi-unitary equivalence}

\subsection{Quasi-unitary equivalence of graph and fractal energy forms}

We come now to the main part of our article, the proof of Theorem 1.1. Let $\mathscr{H}_{m}=\ell_{2}\left(V_{m}, \mu_{m}\right)$ and let $\mathcal{E}_{m}=\mathcal{E}_{G_{m}}$ be the graph energy form as in (1.2) or (3.1). For the limit space, we set $\widetilde{\mathscr{H}}=\mathrm{L}_{2}(K, \mu)$ and $\widetilde{\mathscr{H}^{1}}=\operatorname{dom} \mathcal{E}_{K}$, where $\widetilde{\mathcal{E}}=\mathcal{E}_{K}$ denotes the energy form on the fractal, defined as a limit in (3.3).

We will now show our first main result Theorem 1.1.

Proof of Theorem 1.1. We define the identification operators $J=J_{m}: \mathscr{H}_{m} \longrightarrow \widetilde{\mathscr{H}}$ and $J^{\prime}=J_{m}^{\prime}$ by

$$
J f:=\sum_{x \in V_{m}} f(x) \psi_{x, m} \in \widetilde{\mathscr{H}}, \quad\left(J^{\prime} u\right)(y):=\frac{1}{\mu_{m}(y)}\left\langle u, \psi_{y, m}\right\rangle
$$

for $y \in V_{m}$, then it is easily seen that $J^{*}=J^{\prime}$. Moreover, we let $J^{1} f:=J f$ for $f \in \mathscr{H}_{m}$ and let

$$
J^{\prime 1}: \widetilde{\mathscr{H}^{1}} \longrightarrow \mathscr{H}_{m}^{1} \text { be given by }\left(J^{1} u\right)(x)=u(x),
$$

the evaluation of $u$ in $x \in V_{m} \subset K$.

Note that we have $x \sim_{m} y$ if and only if $\left\langle\psi_{x, m}, \psi_{y, m}\right\rangle \neq 0$ for $x, y \in V_{m}(x \neq y)$, where $\psi_{x, m}$ is the $m$-harmonic function with value 1 at $x \in V_{m}$ and 0 elsewhere. We will make frequent use of the following relation,

$$
\mu_{m}(y)=\int_{K} \psi_{y, m} \mathrm{~d} \mu=\sum_{x \in V_{m}}\left\langle\psi_{x, m}, \psi_{y, m}\right\rangle
$$

which follows from the fact that $\sum_{y \in V_{m}} \psi_{y, m}=\mathbb{1}$. By the Cauchy-Young inequality and 4.1), we have

$$
\begin{aligned}
\|J f\|_{\mathrm{L}_{2}(K, \mu)}^{2} & =\sum_{x, y \in V_{m}} f(x) \overline{f(y)} \underbrace{\left\langle\psi_{x, m}, \psi_{y, m}\right\rangle_{\mathrm{L}_{2}(K, \mu)}}_{\geq 0} \\
& \stackrel{\mathrm{CY}}{\leq} \frac{1}{2} \sum_{x \in V_{m}}|f(x)|^{2} \sum_{y \in V_{m}}\left\langle\psi_{x, m}, \psi_{y, m}\right\rangle+\frac{1}{2} \sum_{y \in V_{m}}|f(y)|^{2} \sum_{x \in V_{m}}\left\langle\psi_{x, m}, \psi_{y, m}\right\rangle \\
& =\sum_{x \in V_{m}}|f(x)|^{2} \underbrace{\left\langle\psi_{x, m}, \mathbb{1}\right\rangle}_{=\mu_{m}(x)}=\|f\|_{\ell_{2}\left(V_{m}, \mu_{m}\right)}^{2},
\end{aligned}
$$

hence we have chosen our vertex weights $\mu_{m}$ on $V_{m}$ appropriately; in particular, the first condition 2.3a of quasi-unitary equivalence is fulfilled as well as the second (recall that $J^{*}=J^{\prime}$ ), both with $\delta=0$.

Let us now check the first estimate in 2.3b): Let $y \in V_{m}$. We apply (4.1) and obtain

$$
\left(f-J^{\prime} J f\right)(y)=\frac{1}{\mu_{m}(y)} \sum_{x \in V_{m}}\left\langle\psi_{x, m}, \psi_{y, m}\right\rangle(f(y)-f(x)) .
$$

Hence, for $f \in \mathscr{H}_{m}$, by the Cauchy-Schwarz inequality,

$$
\begin{aligned}
\left\|f-J^{\prime} J f\right\|_{\ell_{2}\left(V_{m}, \mu_{m}\right)}^{2} & =\sum_{y \in V_{m}} \frac{1}{\mu_{m}(y)}\left|\sum_{x \in V_{m}}\left\langle\psi_{x, m}, \psi_{y, m}\right\rangle(f(y)-f(x))\right|^{2} \\
& \leq \sum_{y \in V_{m}} \frac{1}{\mu_{m}(y)}\left(\sum_{x \in V_{m}} \frac{\left\langle\psi_{x, m}, \psi_{y, m}\right\rangle^{2}}{c_{\{x, y\}, m}}\right) \sum_{x \sim_{m} y} c_{\{x, y\}, m}|f(y)-f(x)|^{2} \\
& \leq \frac{1}{c_{-, m}}\left(\max _{y \in V_{m}} \frac{1}{\mu_{m}(y)} \sum_{x \in V_{m}}\left\langle\psi_{x, m}, \psi_{y, m}\right\rangle^{2}\right) \mathcal{E}_{m}(f)
\end{aligned}
$$


using (3.6) for the last estimate. Now

$$
\max _{y \in V_{m}} \frac{1}{\mu_{m}(y)} \sum_{x \in V_{m}}\left\langle\psi_{x, m}, \psi_{y, m}\right\rangle^{2} \leq \max _{y \in V_{m}} \sum_{x \in V_{m}}\left\langle\psi_{x, m}, \psi_{y, m}\right\rangle=\max _{y \in V_{m}} \mu_{m}(y) \leq \mu_{+, m}
$$

using $\left\langle\psi_{x, m}, \psi_{y, m}\right\rangle \leq\left\langle\mathbb{1}, \psi_{y, m}\right\rangle=\mu_{m}(y)$ for the first inequality, (4.1) for the equality and $\psi_{y, m} \leq 1$ for the last inequality. Moreover, we have estimated $\mu_{m}(y)$ by

$$
\mu_{+, m}:=\max \left\{\max _{x \in V_{m}} \mu_{m}(x), \max _{w \in W_{m}} \mu\left(K_{w}\right)\right\}
$$

(the reason for the second term will become clear in a moment). In particular, the first estimate in $(2.3 \mathrm{~b})$ holds with $\delta=\left(\mu_{+, m} / c_{-, m}\right)^{1 / 2}$.

Next we prove the second inequality of (2.3b); actually, we will show estimate (2.4): We have

$$
u-J J^{\prime 1} u=\sum_{x \in V_{m}}(u-u(x)) \psi_{x, m}=\sum_{w \in W_{m}} \sum_{x \in F_{w}\left(V_{0}\right)}(u-u(x)) \psi_{x, m} \Upsilon_{K_{w}}
$$

almost everywhere for any $u \in \mathscr{H}$ using the fact that $\left\{\psi_{x, m}\right\}_{x \in V_{m}}$ is a partition of unity. In particular, we have

$$
\begin{aligned}
\left\|u-J J^{\prime 1} u\right\|_{\mathrm{L}_{2}(K, \mu)}^{2} & =\sum_{w \in W_{m}}\left\|u-J J^{\prime 1} u\right\|_{\mathrm{L}_{2}\left(K_{w}\right)}^{2} \\
& \leq \sum_{w \in W_{m}} \sum_{x, y \in F_{w}\left(V_{0}\right)}\left\langle\psi_{x, m}, \psi_{y, m}\right\rangle_{\mathrm{L}_{2}\left(K_{w}\right)} \max _{x, z \in K_{w}}|u(x)-u(z)| \max _{y, z \in K_{w}}|u(y)-u(z)| \\
& \leq \frac{1}{c_{-, m}} \sum_{w \in W_{m}} \sum_{x, y \in F_{w}\left(V_{0}\right)}\left\langle\psi_{x, m}, \psi_{y, m}\right\rangle_{\mathrm{L}_{2}\left(K_{w}\right)} \mathcal{E}_{K_{w}}\left(u \uparrow_{K_{w}}\right) \\
& =\frac{1}{c_{-, m}} \sum_{w \in W_{m}} \mu\left(K_{w}\right) \mathcal{E}_{K_{w}}\left(u \uparrow_{K_{w}}\right) \leq \frac{\mu_{+, m}}{c_{-, m}} \widetilde{\mathcal{E}}(u)
\end{aligned}
$$

using Proposition 3.6 for the second inequality, $\sum_{x \in F_{w}\left(V_{0}\right)} \psi_{x, m} \Upsilon_{K_{w}}=\mathbb{1}_{K_{w}}$ for the fourth line and (3.11) resp. (4.2) for the final inequality. In particular, we can again choose $\delta^{\prime}=\left(\mu_{+, m} / c_{-, m}\right)^{1 / 2}$ in (2.4).

For the second estimate in (2.3c) (the first one is trivially fulfilled), let $u \in \mathscr{H}$ and $x \in V_{m}$. Then

$$
\left(J^{\prime} u-J^{\prime 1} u\right)(x)=\frac{1}{\mu_{m}(x)}\left\langle u-u(x) \mathbb{1}, \psi_{x, m}\right\rangle=\sum_{w \in W_{x, m}} \frac{1}{\mu_{m}(x)}\left\langle u-u(x) \mathbb{1}, \psi_{x, m}\right\rangle_{\mathrm{L}_{2}\left(K_{w}\right)},
$$

where $W_{x, m}:=\left\{w \in W_{m} \mid x \in K_{w}\right\}$ are the words whose $m$-cells contain $x$. Hence, we have

$$
\begin{aligned}
\left|\left(J^{\prime} u-J^{\prime 1} u\right)(x)\right|^{2} & \leq\left(\sum_{w \in W_{x, m}} \frac{1}{\mu_{m}(x)} \int_{K_{w}}|u-u(x)| \psi_{x, m} \mathrm{~d} \mu\right)^{2} \\
& \leq \frac{1}{c_{-, m}} \max _{w \in W_{x, m}} \mathcal{E}_{K_{w}}\left(u \uparrow_{K_{w}}\right)\left(\frac{1}{\mu_{m}(x)} \sum_{w \in W_{x, m}} \int_{K_{w}} \psi_{x, m} \mathrm{~d} \mu\right)^{2} \\
& =\frac{1}{c_{-, m}} \max _{w \in W_{x, m}} \mathcal{E}_{K_{w}}\left(u \uparrow_{K_{w}}\right) \leq \frac{1}{c_{-, m}} \sum_{w \in W_{x, m}} \mathcal{E}_{K_{w}}\left(u \uparrow_{K_{w}}\right)
\end{aligned}
$$


using Proposition 3.6 for the second inequality, $\sum_{w \in W_{x, m}} \int_{K_{w}} \psi_{x, m} \mathrm{~d} \mu=\mu_{m}(x)$ for the last line. Now, we obtain for the norm estimate

$$
\begin{aligned}
\left\|J^{\prime} u-J^{\prime 1} u\right\|_{\ell_{2}\left(V_{m}, \mu_{m}\right)}^{2} & \leq \frac{1}{c_{-, m}} \sum_{x \in V_{m}} \sum_{w \in W_{x, m}} \mathcal{E}_{K_{w}}\left(u \uparrow_{K_{w}}\right) \mu_{m}(x) \\
& =\frac{1}{c_{-, m}} \sum_{w \in W_{m}} \mathcal{E}_{K_{w}}\left(u \uparrow_{K_{w}}\right) \sum_{x \in F_{w}\left(V_{0}\right)} \mu_{m}(x) \\
& \leq \frac{N_{0} \mu_{+, m}}{c_{-, m}} \sum_{w \in W_{m}} \mathcal{E}_{K_{w}}\left(u \uparrow_{K_{w}}\right)=\frac{N_{0} \mu_{+, m}}{c_{-, m}} \widetilde{\mathcal{E}}(u)
\end{aligned}
$$

using (4.2) resp. (3.11) for the last line (here $N_{0}=\left|V_{0}\right|$ denotes the number of boundary vertices).

Finally, we check the last condition $(2.3 \mathrm{~d})$ of quasi-unitary equivalence: For any $f=$ $\sum_{x \in V_{m}} f(x) \psi_{x, m} \uparrow_{V_{m}} \in \mathscr{H}_{m}$ and $u \in \mathscr{H}^{1}$, we have

$$
\mathcal{E}_{m}\left(f, J^{\prime 1} u\right)-\widetilde{\mathcal{E}}\left(J^{1} f, u\right)=\sum_{x \in V_{m}} f(x)\left(\mathcal{E}_{m}\left(\psi_{x, m} \uparrow_{V_{m}}, u \uparrow_{V_{m}}\right)-\widetilde{\mathcal{E}}\left(\psi_{x, m}, u\right)\right)=0
$$

using (3.9). We now apply Lemma 2.4 and obtain $(2.3 \mathrm{~b})$ with $\delta_{\mathrm{a}}=0, \delta^{\prime}=\left(\mu_{+, m} / c_{-, m}\right)^{1 / 2}$ and $\delta_{\mathrm{c}}=\left(N_{0} \mu_{+, m} / c_{-, m}\right)^{1 / 2}$. Then $\delta=\delta^{\prime}+\delta_{\mathrm{c}}=\left(1+\sqrt{N_{0}}\right)\left(\mu_{+, m} / c_{-, m}\right)^{1 / 2}$. Collecting all the individual error terms, the quasi-unitary equivalence constant is then

$$
\delta_{m}=\left(1+\sqrt{N_{0}}\right) \cdot\left(\frac{\mu_{+, m}}{c_{-, m}}\right)^{1 / 2}=\frac{1+\sqrt{N_{0}}}{\sqrt{c_{-, 0}}} \cdot\left(r_{+}^{m} \mu_{+, m}\right)^{1 / 2} .
$$

Note that for a general probability measure $\mu$ (not necessarily self-similar), we have at least the estimate $\mu_{+, m} \leq 1$ and hence the following result:

4.1. Corollary. The $\delta_{m}$-quasi-unitary equivalence of the fractal energy form $\mathcal{E}_{K}$ with general Borel regular probability measure $\mu$ of full support and the approximating graph energy forms $\mathcal{E}_{m}=\mathcal{E}_{G_{m}}$ as in Theorem 1.1 holds with

$$
\delta_{m}=\frac{1+\sqrt{N_{0}}}{\sqrt{c_{-, 0}}} \cdot r_{+}^{m / 2}
$$

If the measure is self-similar or if the fractal, its measure and boundary are symmetric, we can obtain better estimates, see Subsection 5.2 .

4.2. Remark. Our results also extends to certain classes of finitely ramified fractals as introduced in [Tep08]: if there is a compatible sequence of energy (or more precisely, resistance) forms on the boundary structure $\left(V_{m}\right)_{m}$. If the diameter of $m$-cells tends to 0 in the resistance metric, and if one chooses a measure such that the measure of $m$-cells tends to 0 , then we obtain a similar result as Theorem 1.1 .

4.3. Remark. Let us comment on the finite element method (FEM) for fractals developed in GRS01, ASST03. In our notation, one can find for example an approximative eigenvalue $\lambda_{m}$ of an eigenvalue $\widetilde{\lambda}$ of $\Delta_{(K, \mu)}$ by finding a non-trivial solution $f \in \ell_{2}\left(V_{m}, \mu_{m}\right)$ (or a vector $\left.f \in \mathbb{C}^{V_{m}}\right)$ of the generalised eigenvalue problem

$$
C_{m} f=\lambda_{m} G_{m} f
$$

where the matrices $C_{m}$ and $G_{m}$ are given by

$$
\left(C_{m}\right)_{x y}=\widetilde{\mathcal{E}}\left(\psi_{x, m}, \psi_{y, m}\right)=\mathcal{E}_{m}\left(\delta_{x}, \delta_{y}\right)= \begin{cases}\sum_{z \sim_{m} x} c_{\{x, z\}, m}, & \text { if } x=y \\ -c_{\{x, y\}, m}, & \text { if } x \sim_{m} y \\ 0, & \text { otherwise }\end{cases}
$$

and

$$
\left(G_{m}\right)_{x y}=\left\langle\psi_{x, m}, \psi_{y, m}\right\rangle_{\widetilde{\mathscr{H}}}
$$


The latter matrix is also called the Gram matrix of $\left(\psi_{x, m}\right)_{x \in V_{m}}$. As a candidate for $f$, one can choose e.g. evaluation of $u$ at the vertices $V_{m}$, i.e., $f=J^{\prime 1} u$. Then $f$ is the coefficient vector of the harmonic interpolation spline $J J^{\prime 1} u=\sum_{x \in V_{m}} f(x) \psi_{x, m}$ in our notation. We show actually as one condition of quasi-unitary equivalence that the harmonic spline $J f=J J^{\prime 1} u$ is close to the original $u$ for large $m \in \mathbb{N}$, see Lemma 2.4 .

\subsection{A direct eigenvalue convergence result}

Although an eigenvalue convergence already follows from quasi-unitary equivalence as in Corollary 1.4, we show a more explicit convergence using Proposition 2.8:

4.4. Theorem. Let $(K, \mu)$ be a pcf fractal with Borel regular probability measure of full support approximable by finite weighted graphs $\left(G_{m}, \mu_{m}\right)$ and let $\lambda_{k}\left(G_{m}, \mu_{m}\right)$ resp. $\lambda_{k}(K, \mu)$ be the $k$-th eigenvalue of the Laplacian operator associated with $\left(G_{m}, \mu_{m}\right)$ resp. $(K, \mu)$ (in increasing order, repeated according to their multiplicity). Then

$$
\frac{1-\delta_{m}}{1+\delta_{m} \lambda_{k,+}} \cdot \lambda_{k}\left(G_{m}, \mu_{m}\right) \leq \lambda_{k}(K, \mu) \leq \frac{1}{1-\delta_{m}\left(1+\lambda_{k,+}\right)} \cdot \lambda_{k}\left(G_{m}, \mu_{m}\right)
$$

provided $m \geq m_{0}$, where $\delta_{m}$ is as in Theorem 1.1 resp. (4.3). Here, $m_{0} \in \mathbb{N}$ is defined by the condition $\delta_{m_{0}}<\left(1+\lambda_{k,+}\right)^{-1}$, where $\lambda_{k}\left(G_{m}, \mu_{m}\right) \leq \lambda_{k,+}$ is an upper bound on the graph eigenvalues.

If $\delta_{m} \leq\left(1+\lambda_{k}(K, \mu)\right)^{-1 / 2}$, then we can choose as upper bound

$$
\lambda_{k}\left(G_{m}, \mu_{m}\right) \leq 2 \lambda_{k}(K, \mu)=: \lambda_{k,+} .
$$

Proof. We apply Proposition 2.8 with $\mathscr{H}=\ell_{2}\left(V_{m}, \mu_{m}\right)$ and $\widetilde{\mathscr{H}}=\mathrm{L}_{2}(K, \mu)$ etc. We note first that (2.9a) is trivially fulfilled (even with equality) as

$$
\left(J^{1} J^{1} f\right)(y)=\sum_{x \in V_{m}} f(x) \psi_{x, m}(y)=f(y) .
$$

In particular, the upper estimate on $\lambda_{k}(K, \mu)$ in (4.4) holds (using the second inequality in $(2.9 \mathrm{c})$ ).

For the lower bound (4.4), we note that $2.9 \mathrm{~b})$ is fulfilled as

$$
\widetilde{\mathcal{E}}\left(u, J^{1} J^{\prime 1} u\right)=\mathcal{E}_{m}\left(u \uparrow_{V_{m}},\left(J^{1} J^{\prime 1} u\right) \uparrow_{V_{m}}\right)=\mathcal{E}_{m}\left(u \uparrow_{V_{m}}, u \uparrow_{V_{m}}\right) \leq \widetilde{\mathcal{E}}(u, u)
$$

using (3.9) and (3.8).

For the upper bound in 4.5 we use the first inequality in $2.9 \mathrm{c}$.

\subsection{Eigenvalue estimates for subsequent graphs}

If the measure $\mu$ is self-similar (see Subsection 5.1), then we can also show that the $k$-th eigenvalue of the graph energy forms $\mathcal{E}_{m}$ and $\mathcal{E}_{m+1}$ are close to each other. Denote by $\lambda_{k}\left(G_{m}, \mu_{m}\right)$ the $k$-th eigenvalue of the graph Laplacian $\Delta_{m}$ associated with $\mathcal{E}_{m}$ (in increasing order, repeated according to the multiplicity). Blasiak, Strichartz and Uguurcan [BSU08, text after eq. (2.9)] believe that the sequence $\left(\lambda_{k}\left(G_{m}, \mu_{m}\right)\right)_{m \in \mathbb{N}}$ is monotonely increasing. We can at least show the following:

4.5. Proposition. Assume that the measure $\mu$ on $K$ is self-similar, then we have

$$
\lambda_{k}\left(G_{m}, \mu_{m}\right) \leq \frac{1}{1-\frac{\lambda_{k,+}}{\lambda_{1}^{\mathrm{D}}} \cdot\left(\mu_{+} r_{-}\right)^{m}} \cdot \lambda_{k}\left(G_{m+1}, \mu_{m+1}\right)
$$

where $\lambda_{1}^{\mathrm{D}}>0$ is the smallest eigenvalue of the Dirichlet Laplacian (the Laplacian $\Delta_{\left(G_{1}, \mu_{1}\right)}$ restricted to $\left.V_{1} \backslash V_{0}\right)$ and where $\lambda_{k,+}$ is an upper bound on $\lambda_{k}\left(G_{m+1}, \mu_{m+1}\right)$. 
Proof. We apply Proposition 2.7 with

$$
J=J_{m+1, m}: \mathscr{H}_{m+1}=\ell_{2}\left(V_{m+1}, \mu_{m+1}\right) \longrightarrow \mathscr{H}_{m}=\ell_{2}\left(V_{m}, \mu_{m}\right), \quad J u:=u \uparrow_{V_{m}},
$$

then we have

$$
\begin{aligned}
\|J u\|_{\ell_{2}\left(V_{m}, \mu_{m}\right)}^{2}-\|u\|_{\ell_{2}\left(V_{m+1}, \mu_{m+1}\right)}^{2} & =\sum_{x \in V_{m}}|u(x)|^{2}\left(\mu_{m}(x)-\mu_{m+1}(x)\right)-\sum_{x \in V_{m+1} \backslash V_{m}}|u(x)|^{2} \mu_{m+1}(x) \\
& \geq\|u\|_{\ell_{2}\left(V_{m}, \mu_{m}\right)}^{2}\left(1-\mu_{-}\right)-\|u\|_{\ell_{2}\left(V_{m+1} \backslash V_{m}, \mu_{m+1}\right)}^{2} \\
& \geq-\|u\|_{\ell_{2}\left(V_{m+1} \backslash V_{m}, \mu_{m+1}\right)}^{2}
\end{aligned}
$$

as $\mu_{m+1}(x) \geq \mu_{-} \mu_{m}(x)$ for $x \in V_{m}$ (using (5.2p).

We can consider $\Delta_{\left(G_{m+1}, \mu_{m+1}\right)}$ restricted to $\ell_{2}\left(V_{m+1} \backslash V_{m}, \mu_{m+1}\right)$ as Dirichlet Laplacian (denoted by $\left.\Delta_{\left(G_{m+1}, \mu_{m+1}\right)}^{\mathrm{D}}\right)$ with Dirichlet boundary condition on the "boundary" $V_{m} \subset V_{m+1}$ (see e.g. [Pos16, Sec. 6.7]). We then have (using a variant of the min-max characterisation of eigenvalues)

$$
\lambda_{1}\left(\Delta_{\left(G_{m+1}, \mu_{m+1}\right)}^{\mathrm{D}}\right) \leq \frac{\mathcal{E}_{m+1}(u)}{\|u\|_{\ell_{2}\left(V_{m+1} \backslash V_{m}, \mu_{m+1}\right)}^{2}}
$$

for any $u: V_{m+1} \backslash V_{m} \longrightarrow \mathbb{C}$. Note that a Dirichlet eigenvalue is always positive.

As the Dirichlet Laplacian on $V_{m+1} \backslash V_{m}$ is a direct sum of a rescaled copy of the one on $V_{1} \backslash V_{0}$, we can estimate the first eigenvalue on generation $m+1$ by

$$
\begin{aligned}
\lambda_{1}\left(\Delta_{\left(G_{m+1}, \mu_{m+1}\right)}^{\mathrm{D}}\right) & =\inf _{u} \frac{\mathcal{E}_{m+1}(u)}{\|u\|_{\ell_{2}\left(V_{m+1} \backslash V_{m}, \mu_{m+1}\right)}^{2}} \\
& \geq \frac{1}{\left(r_{+} \mu_{-}\right)^{m}} \inf _{u_{1}} \frac{\mathcal{E}_{1}(u)}{\|u\|_{\ell_{2}\left(V_{1} \backslash V_{0}, \mu_{1}\right)}^{2}}=\frac{1}{\left(r_{+} \mu_{-}\right)^{m}} \overbrace{\lambda_{1}\left(\Delta_{\left(G_{1}, \mu_{1}\right)}^{\mathrm{D}}\right)}^{=: \lambda_{1}^{\mathrm{D}}}
\end{aligned}
$$

using (3.5), (3.6) and (5.1) (as $\mu_{-}$is the minimum of the measure scaling factors $\mu_{j}$ ). We conclude from (4.6) and the Dirichlet eigenvalue estimates (4.7)-(4.8) that

$$
\|J u\|_{\ell_{2}\left(V_{m}, \mu_{m}\right)}^{2}-\|u\|_{\ell_{2}\left(V_{m+1}, \mu_{m+1}\right)}^{2} \geq-\frac{1}{\lambda_{1}\left(\Delta_{\left(G_{m+1}, \mu_{m+1}\right)}^{\mathrm{D}}\right)} \mathcal{E}_{m+1}(u) \geq-\frac{\left(r_{+} \mu_{-}\right)^{m}}{\lambda_{1}^{\mathrm{D}}} \mathcal{E}_{m+1}(u),
$$

and we can choose $\delta_{1}=\left(r_{+} \mu_{-}\right)^{m} / \lambda_{1}^{\mathrm{D}}$ in Proposition 2.7. As $\mathcal{E}_{m}\left(u \uparrow_{V_{m}}\right) \leq \mathcal{E}_{m+1}(u)$ by the compatibility of $\left(\mathcal{E}_{G_{m}}\right)_{m}$ the condition on the energy forms in Proposition 2.7 is also fulfilled. The result now follows from Proposition 2.7.

4.6. Remark. If the fractal is symmetric with symmetric measure (see Subsection 5.2), then $\left(\mu_{+} r_{-}\right)^{m}=\left(r_{0} / N\right)^{m}$, and the factor is of the form $1+\mathrm{O}\left(\left(r_{0} / N\right)^{m}\right)$, depending on the a priori bound $\lambda_{k,+}$ on $\lambda_{k}\left(G_{m+1}, \mu_{m+1}\right)$, hence the sequence $\left(\lambda_{k}\left(G_{m}, \mu_{m}\right)\right)_{m}$ is close to a monotonely increasing sequence at least for large $m$.

\section{Examples}

In this section we present some classes of examples and some concrete ones. Note that the contraction ratio $\theta_{j}$ does not play any role in the convergence results.

\subsection{Self-similar and other measures on pcf fractals}

A probability measure $\mu$ on $K$ is called self-similar, if $\mu$ is a Borel regular probability measure and if there are so-called measure scaling parameters $\mu_{1}, \ldots, \mu_{N}>0$ such that

$$
\mu(A)=\sum_{j=1}^{N} \mu_{j} \mu\left(F_{j}^{-1}(A)\right)
$$


for all Borel sets $A \subset K$. Note that a self-similar measure always exists for any fractal $K$ defined via a (finite) IFS. In particular, an $m$-cell $K_{w}=F_{w}(K)$ has measure

$$
\mu\left(K_{w}\right)=\mu_{w_{1}} \cdot \ldots \cdot \mu_{w_{m}},
$$

hence

$$
\mu_{m,-}=\left(\mu_{-}\right)^{m}, \quad \text { where } \quad \mu_{-}:=\min \left\{\mu_{1}, \ldots, \mu_{N}\right\} \in(0,1) .
$$

One choice of measure is given by

$$
\mu_{j}=r_{j}^{d}
$$

where $d$ is defined by $\sum_{j=1}^{N} r_{j}^{d}=1$. Here, $d$ is the Hausdorff dimension of $(K, \varrho)$, cf. Kig01, Thm. 4.2.1], where $\varrho$ is the resistance metric. If the embedding space $X=\mathbb{R}^{d}$ is the Euclidean space, then we can also calculate the Hausdorff dimension $d_{\text {eucl }}$ of $K$ with respect to the Euclidean metric: the number $d_{\text {eucl }}$ is defined via $\sum_{j=1}^{N} \theta_{j}^{d_{\text {eucl }}}=1$ for self-similar sets satisfying the so-called open set condition (see e.g. [Kig01, Sec. 1.5]). Here, $\theta_{j}$ is the contraction ratio of the contractive similarity $F_{j}: \mathbb{R}^{d} \longrightarrow \mathbb{R}^{d}$.

There is another natural measure on a fractal approximable by finite weighted graphs, namely the Kusuoka energy measure. For a definition, see e.g. [Tep08, Def. 3.4]. A good reference and a link to the unusual properties of the domain of the Laplacian on a fractal can be found in [BBST99]. There is even an explicit formula (cf. [Tep08, Thm. 6.1]) for the Laplacian $\Delta_{(K, \mu)}$, if $\mu$ is the Kusuoka energy measure resembling the Laplacian of a Riemannian manifold (normalised to $\mu(K)=1$ ). Theorem 1.1 applies also to this setting; one just needs to calculate $\mu_{m}(x)=\int_{K} \psi_{x, m} \mathrm{~d} \mu$ with respect to this measure.

\subsection{Symmetric pcf fractals}

\subsection{Definition.}

(i) We call a self-similar measure $\mu$ symmetric or homogeneous if the measure rescaling parameters are all the same, namely

$$
\mu_{j}=\frac{1}{N}
$$

for $j=1, \ldots, N$.

(ii) We say that a fractal $K$ approximable by finite weighted graphs is symmetric, if all contraction ratios $\theta_{j}$ of the IFS are the same and if all energy renormalisation parameters $r_{j}$ of the energy forms are the same, i.e., there exists $\theta_{0}, r_{0} \in(0,1)$ such that

$$
\theta_{0}=\theta_{j} \quad \text { and } \quad r_{0}=r_{j},
$$

for all $j=1, \ldots, N$.

(iii) We say that $(K, \mu)$ is symmetric if $K$ is a symmetric fractal together with a symmetric self-similar measure $\mu$.

(iv) Let $V_{0} \subset K$ be the boundary of a fractal $K$ approximable by finite weighted graphs. We say that $V_{0}$ is symmetric, if the weights $\mu_{0}\left(x_{0}\right)=\int_{K} \psi_{x_{0}, 0} \mathrm{~d} \mu$ are all the same, namely

$$
\mu_{0}\left(x_{0}\right)=\frac{1}{N_{0}}
$$

for all $x_{0} \in V_{0}$ (recall that $N_{0}:=\left|V_{0}\right| \leq N$ and that $\left.\sum_{x_{0} \in V_{0}} \mu_{0}\left(x_{0}\right)=\mu(K)=1\right)$.

For a symmetric fractal $K$, the Hausdorff dimension of $K$ with respect to the resistance metric is given by $d=-\log N / \log r_{0}>0$, see e.g. [Kig01, Thm. 4.2.1]. Moreover, the Hausdorff dimension of $K$ with respect to the induced metric from the Euclidean ambient space is $d_{\text {eucl }}=$ $-\log N / \log \theta_{0}>0$ (see [Kig01, Sec. 1.5]). 
Recall that $W_{x, m}=\left\{w \in W_{m} \mid x \in K_{w}\right\}$ denotes the set of words $w$ such that the corresponding cells $K_{w}$ meet in the vertex $x \in V_{m}$. Denote by

$$
N_{1}:=\max _{x \in V_{m}}\left|W_{x, m}\right|
$$

the maximal number of cells meeting in one vertex (note that we have $N_{1} \leq N_{0} \leq N$ ). We can now estimate the weights $\mu_{m}(x)$ and $\mu_{+, m}$ :

5.2. Lemma. Let $K$ be a symmetric fractal and $\mu$ be a symmetric self-similar measure.

(i) All m-cells $K_{w}$ have the same measure, namely

$$
\mu\left(K_{w}\right)=\frac{1}{N^{m}}
$$

for all $w \in W_{m}$.

(ii) The vertex measure $\mu_{m}(x)$ fulfils

$$
\mu_{m}(x) \leq \frac{\left|W_{x, m}\right|}{N^{m}} \leq \frac{N_{1}}{N^{m}} \leq \frac{1}{N^{m-1}} .
$$

(iii) If, in addition, the boundary $V_{0}$ of $K$ is symmetric, then we have

$$
\mu_{m}(x)=\frac{\left|W_{x, m}\right|}{N_{0} N^{m}} \leq \frac{N_{1}}{N_{0} N^{m}} \leq \frac{1}{N^{m}}
$$

Proof. Eq. (5.3a) follows from the scaling property (5.1) $\mu$ with $\mu_{j}=1 / N$. Eq. (5.3b) is a direct consequence using the estimate $\psi_{x, m} \leq 1$ and the fact that $\operatorname{supp} \psi_{x, m}=\bigcup_{w \in W_{x, m}} K_{w}$. Finally, (5.3c) follows again by the scaling property (5.1).

We immediately conclude together with Theorem 1.1 .

5.3. Corollary. Assume that $K$ is a symmetric fractal with symmetric self-similar measure $\mu$, then the $\delta_{m}$-quasi-unitary equivalence of the fractal energy form $\mathcal{E}_{K}$ and the approximating graph energy forms $\mathcal{E}_{m}=\mathcal{E}_{G_{m}}$ as in Theorem 1.1 holds with

$$
\delta_{m}=\frac{\left(1+\sqrt{N_{0}}\right) \sqrt{N_{1}}}{\sqrt{c_{-, 0}}} \cdot\left(\frac{r_{0}}{N}\right)^{m / 2} .
$$

If in addition, the boundary $V_{0}$ of $K$ is also symmetric, then Theorem 1.1 holds with

$$
\delta_{m}=\frac{\left(1+\sqrt{N_{0}}\right) \sqrt{N_{1}}}{\sqrt{c_{-, 0} N_{0}}} \cdot\left(\frac{r_{0}}{N}\right)^{m / 2} .
$$

Proof. Recall the definition of $\mu_{+, m}$ in 4.2 . From Lemma 5.2 we conclude

$$
\mu_{+, m}=\frac{N_{1}}{N^{m}} \leq \frac{1}{N^{m-1}} \quad \text { resp. } \quad \mu_{+, m}=\frac{N_{1}}{N_{0} N^{m}} \leq \frac{1}{N^{m}}
$$

in the case of a non-symmetric resp. symmetric boundary, hence the result follows from the expression of $\delta_{m}$ in 4.3 for Theorem 1.1 .

For a symmetric fractal $K$, the number of vertices $\left|V_{m}\right|$ is given recursively by $\left|V_{0}\right|=N_{0}$ and $\left|V_{m+1}\right|:=N\left|V_{m}\right|-b$, where $b$ is the number of vertices identified from one generation to the next one. In particular, we have

$$
\left|V_{0}\right|=N_{0}, \quad\left|V_{m}\right|=N^{m} N_{0}-\frac{N^{m}-1}{N-1} b,
$$

and $\left|V_{m}\right|=N^{m} N_{0}+\mathrm{O}\left(N^{m-1}\right)$ if $m \rightarrow \infty$, i.e., the size of the approximating matrices of $\Delta_{\left(G_{m}, \mu_{m}\right)}$ increases of order $N^{m} N_{0}$ as $m \rightarrow \infty$. 


\subsection{Sierpiński gasket and related fractals}

The unit interval. The unit interval $K=[0,1]$ can be seen as a self-similar fractal with $F_{1}(x)=x / 2$ and $F_{2}(x)=x / 2+1 / 2$ with boundary $V_{0}=\{0,1\}$. This fractal is symmetric, and if we choose the symmetric self-similar measure $\mu$ (which is here the one-dimensional Lebesgue measure). In particular, $K, \mu$ and $V_{0}$ are all symmetric and we have

$$
N=N_{0}=N_{1}=2, \quad \theta_{0}=\frac{1}{2}, \quad r_{0}=\frac{1}{2}, \quad b=1, \quad\left|V_{m}\right|=2^{m}+1 .
$$

Moreover, the conductances at generation 0 are $c_{e_{0}}=1$ for the single edge $e_{0}$ in $G_{0}$. The approximating graphs $G_{m}$ are path graphs with $2^{m}+1$ vertices. The error is then given by

$$
\delta_{m}=(1+\sqrt{2}) \cdot \frac{1}{2^{m}} .
$$

This convergence rate is quite good, as $\delta_{m}$ is smaller than 0.01 for $m \geq 8$, and the number of vertices is still not too large, namely $\left|V_{8}\right|=2^{8}+1=257$.

Sierpiński gasket. The Sierpiński gasket is given by three contractions with ratio $\theta_{0}=1 / 2$ and fixed points at the vertices of an equilateral triangle. Here, $N=N_{0}=3$ and $N_{1}=2$. Again, this fractal is symmetric and we fix the symmetric measure $\mu$; also the boundary is symmetric. In particular, we have

$$
N=N_{0}=3, \quad N_{1}=2, \quad \theta_{0}=\frac{1}{2}, \quad r_{0}=\frac{3}{5}, \quad b=3, \quad\left|V_{m}\right|=\frac{3}{2}\left(3^{m}+1\right) .
$$

Moreover, we have $c_{e_{0}}=1$; and the error is given by

$$
\delta_{m}=\frac{(1+\sqrt{3}) \sqrt{2}}{\sqrt{3}} \cdot \frac{1}{5^{m / 2}} .
$$

The error $\delta_{m}$ is smaller than 0.01 for $m \geq 7$, but the number of vertices is then already quite large, namely $\left|V_{7}\right|=3282$.

Sierpiński gaskets in higher dimension. Here, we consider the self-similar symmetric set in $\mathbb{R}^{N-1}$ for $N \geq 2$ with contraction ratio $\theta_{0}=1 / 2$ and $N$ fixed points lying on an $N$-dimensional pyramid with side length 1 . For $N=2$, this is the interval, for $N=3$ the fractal is the Sierpiński gasket and for $N=4$ the Sierpiński pyramid. We also have $N_{0}=N$ and $N_{1}=2$ (at most two cells meet). Also the boundary is symmetric. We have here

$$
N=N_{0}, \quad N_{1}=2, \quad \theta_{0}=\frac{1}{2}, \quad r_{0}=\frac{N}{N+2}, \quad b=\frac{N(N-1)}{2}, \quad\left|V_{m}\right|=\frac{N}{2}\left(N^{m}+1\right) .
$$

Moreover, we have $c_{e_{0}}=1$; and the error is given by

$$
\delta_{m}=\frac{(1+\sqrt{N}) \sqrt{2}}{\sqrt{N}} \cdot \frac{1}{(N+2)^{m / 2}} .
$$

If e.g. $N=4$, we have $\delta_{m}$ smaller than 0.01 for $m \geq 6$, but the number of vertices is then already quite large, namely $\left|V_{6}\right|=8194$.

Related fractals. The level $n$ Sierpiński gasket $S G_{n}$ is a self-similar compact set in $\mathbb{R}^{2}$ defined as follows (see e.g. [Str06, Ex. 4.1.1, Fig. 4.1.1]): subdivide an equilateral triangle into $3(n-1)$ equilateral triangles of side length $\theta_{0}=\theta_{j}=1 / n$ of the original side length. Each of these $3(n-1)$ triangles can be obtained by a similarity from the original triangle with contraction ratio $1 / n$ and a fixed point. Hence, we have $N=3(n-1)$ fixed points, and only the 3 fixed points on the vertices of the original triangle form the boundary, hence $N_{0}=3$. By $D_{3}$-symmetry, we choose all conductances $c_{e_{0}}$ to be equal, say 1 . The renormalisation factors are again all the same and given e.g. for $n=3$ by $r_{0}=7 / 15$ (see [Str06, Ex. 4.3.1]). The level $n$ Sierpiński gasket is an example of a fractal where up to 3 cells meet at a junction point (if 
$n=3$, it is the junction point in the centre of a cell in the previous generation), hence $N_{1}=3$. For the level 3 Sierpiński gasket, we have

$$
N=6, \quad N_{0}=N_{1}=3, \quad \theta_{0}=\frac{1}{2}, \quad r_{0}=\frac{7}{15}, \quad b=8, \quad\left|V_{m}\right|=\frac{7}{5} \cdot 6^{m}+\frac{8}{5} .
$$

Moreover, we can choose $c_{e_{0}}=1$ for all three edges of $G_{0}$; and the error is given by

$$
\delta_{m}=\frac{(1+\sqrt{3}) \sqrt{3}}{\sqrt{3}}\left(\frac{7 / 15}{6}\right)^{m / 2}=(1+\sqrt{3})\left(\frac{7}{90}\right)^{m / 2} .
$$

The error $\delta_{m}$ is smaller than 0.01 for $m \geq 5$, but the number of vertices is then already quite large, namely $\left|V_{5}\right|=10888$.

\subsection{Pentagasket}

The pentagasket is the self-similar structure with five similarities of contraction ratio $\theta_{0}=$ $(3-\sqrt{5}) / 2 \approx 0.382$ and fixed points located at the vertices of a pentagon (see [ASST03] or [Str06, Example 4.3.3 and Exercise 4.3.2]).

One can start with all five vertices as $V_{0}$, hence $G_{0}$ is the complete graph $K_{5}$ with $\left|E_{0}\right|=10$ edges (not embeddable in the plane), cf. Figure 1. Here, we have $N=N_{0}=5$, and at most $N_{1}=2$ cells meet in one vertex. Moreover,

$$
\theta_{0}=\frac{3-\sqrt{5}}{2} \approx 0.382, \quad r_{0}=\frac{\sqrt{161}-9}{8} \approx 0.461, \quad b=5, \quad\left|V_{m}\right|=\frac{15}{4} \cdot 5^{m}+\frac{5}{4} .
$$

Moreover, the conductances of the 10 edges at generation 0 can be chosen to be

$$
c_{e_{0}}= \begin{cases}\frac{\sqrt{161}-7}{16} \approx 0.356 & \text { if } e_{0} \text { is on the pentagon } \\ \frac{15-\sqrt{161}}{16} \approx 0.144 & \text { if } e_{0} \text { joins every second vertex }\end{cases}
$$

By symmetry, the five elementary harmonic functions on $K$ all have the same integral value $\int_{K} \psi_{x_{0}, 0} \mathrm{~d} \mu=1 / 5$ for $x_{0} \in V_{0}$, hence $\mu_{m}(x)=2 / 5^{m+1}$ if $x$ is a junction point and $\mu_{m}(x)=$ $1 / 5^{m+1}$ if not. Moreover, any $m$-cells has measure $1 / 5^{m}$, hence $\mu_{+, m}=1 / 5^{m}$.

The error is then given by

$$
\delta_{m}=\frac{(1+\sqrt{5}) \sqrt{2}}{\left(c_{-, 0}\right)^{1 / 2} \sqrt{5}} \cdot\left(\frac{\sqrt{161}-9}{5 \cdot 8}\right)^{m / 2} \leq 5.3848 \cdot 0.3037^{m}
$$

see 4.3$)$. Here, $q:=((\sqrt{161}-9) / 40)^{1 / 2}=0.30366 \ldots \leq 0.3037$; so $q^{m}$ is smaller than 0.01 for $m \geq 6$, and the number of vertices is then $\left|V_{6}\right|=58595$.

A fractal with non-symmetric boundary. If we start with only three boundary points $V_{0}$ (two opposite of the third on the pentagon), and if one uses the five similarities first contracting towards the fixed point and then rotating around the centre of the pentagon by $2 \pi / 5$, then one obtains another approximating sequence for the pentagasket, see Figure 2. Here
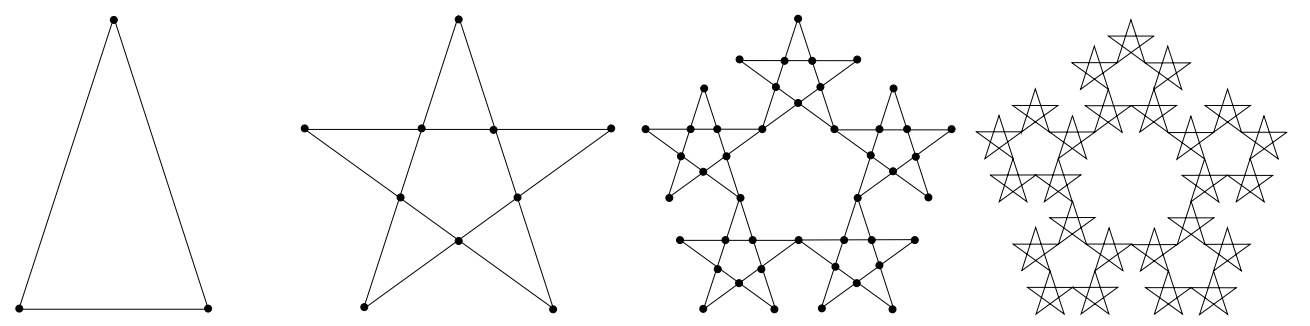

FiguRE 2. The pentagasket approximation graphs $G_{m}$ starting with only three boundary vertices for $m=0, \ldots, 3$ with $\left(\left|V_{m}\right|\right)_{m}=(3,10,10 \cdot 5-5=45,45 \cdot 5-5=$ $220, \ldots)$. 


$$
c_{e_{0}}= \begin{cases}\frac{\sqrt{161}-7}{14} \approx 0.406 & \text { if } e_{0} \text { is the base (shorter side) of } G_{0} \\ \frac{21-\sqrt{161}}{28} \approx 0.297 & \text { if } e_{0} \text { is one of the sides of } G_{0} .\end{cases}
$$

Note that $G_{0}$ is an isosceles triangle, hence the fractal has no longer a symmetric boundary (as the rotational symmetry of $G_{0}$ is no longer present $)$. The renormalisation factor $r_{0}=(\sqrt{161}-$ $9) / 8$ is the same as above, only $N_{0}=3$ and $\left|V_{m}\right|=\frac{7}{4} \cdot 5^{m}+\frac{5}{4}$ changes from the above setting, hence the error term $\delta_{m}$ is the same as above, just with the factor $(1+\sqrt{5}) \sqrt{2} /\left(\left(c_{-, 0}\right)^{1 / 2} \sqrt{5}\right) \leq$ 5.3848 replaced by $(1+\sqrt{3}) \sqrt{2} /\left(c_{-, 0}\right)^{1 / 2} \leq 7.0917$. Moreover, $\delta_{m}$ is smaller than 0.01 if $m \geq 6$, and here $\left|V_{6}\right|=27345$.

\section{References}

[ASST03] B. Adams, S. A. Smith, R. S. Strichartz, and A. Teplyaev, The spectrum of the Laplacian on the pentagasket, Fractals in Graz 2001, Trends Math., Birkhäuser, Basel, 2003, pp. 1-24.

[BBST99] O. Ben-Bassat, R. S. Strichartz, and A. Teplyaev, What is not in the domain of the Laplacian on Sierpinski gasket type fractals, J. Funct. Anal. 166 (1999), 197-217.

$\left[\mathrm{BCH}^{+} 17\right]$ A. Brzoska, A. Coffey, M. Hansalik, S. Loew, and L. G. Rogers, Spectra of magnetic operators on the diamond lattice fractal, arXiv:1704.01609 (2017).

[BHS09] T. Berry, S. M. Heilman, and R. S. Strichartz, Outer approximation of the spectrum of a fractal Laplacian, Experiment. Math. 18 (2009), 449-480.

[BSU08] A. Blasiak, R. S. Strichartz, and B. E. Uğurcan, Spectra of self-similar Laplacians on the Sierpinski gasket with twists, Fractals 16 (2008), 43-68.

[DCR18] P. Dondl, K. Cherednichenko, and F. Rösler, Norm-resolvent convergence in perforated domains, to appear in Asymptot. Anal. (2018).

[FS92] M. Fukushima and T. Shima, On a spectral analysis for the Sierpiński gasket, Potential Anal. 1 (1992), 1-35.

[GRS01] M. Gibbons, A. Raj, and R. S. Strichartz, The finite element method on the Sierpinski gasket, Constr. Approx. 17 (2001), 561-588.

$\left[\mathrm{HKM}^{+} 17\right]$ J. Hyde, D. Kelleher, J. Moeller, L. Rogers, and L. Seda, Magnetic Laplacians of locally exact forms on the Sierpinski Gasket, Commun. Pure Appl. Anal. 16 (2017), 2299-2319.

[HR16] M. Hinz and L. Rogers, Magnetic fields on resistance spaces, J. Fractal Geom. 3 (2016), 75-93.

[HT13] M. Hinz and A. Teplyaev, Vector analysis on fractals and applications, Fractal geometry and dynamical systems in pure and applied mathematics. II. Fractals in applied mathematics, Contemp. Math., vol. 601, Amer. Math. Soc., Providence, RI, 2013, pp. 147-163.

[HT15]_ Closability, regularity, and approximation by graphs for separable bilinear forms, Zap. Nauchn. Sem. S.-Peterburg. Otdel. Mat. Inst. Steklov. (POMI) 441 (2015), 299-317.

$\left[\mathrm{IPR}^{+} 10\right]$ M. Ionescu, E. P. J. Pearse, L. G. Rogers, H.-J. Ruan, and R. S. Strichartz, The resolvent kernel for PCF self-similar fractals, Trans. Amer. Math. Soc. 362 (2010), 4451-4479.

[Kat66] T. Kato, Perturbation theory for linear operators, Springer-Verlag, Berlin, 1966.

[Kig93a] J. Kigami, Harmonic calculus on p.c.f. self-similar sets, Trans. Amer. Math. Soc. 335 (1993), $721-755$.

[Kig93b] _ Harmonic metric and Dirichlet form on the Sierpiński gasket, Asymptotic problems in probability theory: stochastic models and diffusions on fractals (Sanda/Kyoto, 1990), Pitman Res. Notes Math. Ser., vol. 283, Longman Sci. Tech., Harlow, 1993, pp. 201-218.

[Kig01] _ Analysis on fractals, Cambridge Tracts in Mathematics, vol. 143, Cambridge University Press, Cambridge, 2001.

[KP18] A. Khrabustovskyi and O. Post, Operator estimates for the crushed ice problem, to appear in Asymptot. Anal. (2018).

[KS03] K. Kuwae and T. Shioya, Convergence of spectral structures: a functional analytic theory and its applications to spectral geometry, Comm. Anal. Geom. 11 (2003), 599-673.

[Mos94] U. Mosco, Composite media and asymptotic Dirichlet forms, J. Funct. Anal. 123 (1994), 368-421.

[Pos06] O. Post, Spectral convergence of quasi-one-dimensional spaces, Ann. Henri Poincaré 7 (2006), 933973.

[Pos12] _ Spectral analysis on graph-like spaces, Lecture Notes in Mathematics, vol. 2039, Springer, Heidelberg, 2012.

[Pos16]__ Boundary pairs associated with quadratic forms, Math. Nachr. 289 (2016), 1052-1099. 
[PS18] O. Post and J. Simmer, Approximation of fractals by manifolds and other graph-like spaces, (arXiv:1802.02998) (2018).

[RS80] M. Reed and B. Simon, Methods of modern mathematical physics I: Functional analysis, Academic Press, New York, 1980.

[Shi96] T. Shima, On eigenvalue problems for Laplacians on p.c.f. self-similar sets, Japan J. Indust. Appl. Math. 13 (1996), 1-23.

[Str03] R. S. Strichartz, Fractafolds based on the Sierpinsski gasket and their spectra, Trans. Amer. Math. Soc. 355 (2003), 4019-4043.

[Str06] _ Differential equations on fractals, Princeton University Press, Princeton, NJ, 2006.

[SU00] R. S. Strichartz and M. Usher, Splines on fractals, Math. Proc. Cambridge Philos. Soc. 129 (2000), 331-360.

[Tep08] A. Teplyaev, Harmonic coordinates on fractals with finitely ramified cell structure, Canad. J. Math. 60 (2008), 457-480. 